\title{
The Role of Preconditioning in the Evolution of Open-Ocean Deep Convection
}

\author{
Yign NoH AND Woo Geun CheON \\ Department of Atmospheric Sciences, Yonsei University, Seoul, Korea \\ SIEGFRIED RAASCH \\ Institute of Meteorology and Climatology, University of Hannover, Hannover, Germany
}

(Manuscript received 3 January 2002, in final form 12 November 2002)

\section{ABSTRACT}

\begin{abstract}
Large-eddy simulation of open-ocean deep convection shows that the evolution of convection appears in a fundamentally different pattern, depending on the precondition of the ocean and the magnitude of the surface buoyancy flux. As the intensity of the cyclonic gyre in the ocean under the cooling increases, the pattern of convection is transformed from "distributed convection" to "localized convection." In localized convection the typical pattern of open-ocean deep convection appears, such as the generation of baroclinic instability and large lateral buoyancy transfer, secondary circulation, restratification, and the breakup of the original cyclonic gyre. On the other hand, in distributed convection small-scale convective plumes appear uniformly over the whole surface similarly to the convective boundary layer without generating the typical features of open-ocean deep convection. Hence, an enormous difference in the generated eddy kinetic energies exists between the two cases. It is also found that a stronger cooling at the sea surface suppresses the transition from distributed convection to localized convection. Dimensional analysis provides the parameters to characterize the pattern of convection, and the critical condition for the transition is estimated by analyzing the numerical results.
\end{abstract}

\section{Introduction}

It is well established that the onset of open-ocean deep convection requires a cyclonic gyre of the horizontal scale of 50-200 km, which leads to the doming of isopycnals and thus brings weakly stratified waters of the deeper ocean close to the surface (see, e.g., Marshall and Schott 1999; Send and Käse 1998; Schott et al. 1994). Vigorous buoyancy loss during the cooling event, such as the strong wind outburst of continental air in the winter, initiates the deep convection of the very weakly stratified exposed water mass in the core of the cyclonic gyre. Numerous downward plumes of the horizontal scale of $1 \mathrm{~km}$ are generated and penetrated downward while causing violent vertical mixing. Subsequently, baroclinic eddies of the horizontal scale of $10 \mathrm{~km}$ are generated along the cyclonic gyre and transfer heat laterally outward from the gyre. All of these processes suggest that the precondition of the ocean plays an important role during open-ocean deep convection.

Meanwhile, most numerical and laboratory experiments of open-ocean deep convection have been carried out in the form of so-called disk-cooling experiments

Corresponding author address: Yign Noh, Department of Atmospheric Sciences, Yonsei University, 134 Shinchon-dong, Seodaemun-gu, Seoul 120-749, Korea.

E-mail: noh@atmos.yonsei.ac.kr in which the localized cooling over a disk-shaped area is applied on the surface of the initially horizontally homogeneous ocean (i.e., Jones and Marshall 1993; Madec et al. 1991; Maxworthy and Narimousa 1994; Sander et al. 1995; Coates et al. 1995; Visbeck et al. 1996; Whitehead et al. 1996; Raasch and Etling 1998; Noh et al. 1999). In these experiments the radial buoyancy gradient across the rim of a convective column under the disk-shaped cooling, or a chimney, increases with time. The radial buoyancy gradient is balanced by a geostrophic rim current around the convective column. As the radial buoyancy gradient increases with time, the intensity of the rim current increases accordingly and the flow becomes baroclinically unstable. Baroclinic eddies develop along it and detach themselves from the rim current ultimately. These baroclinic eddies are responsible for the lateral transfer of heat to the outside of the convective column. Visbeck et al. (1996) suggested that an equilibrium state is reached with time between the lateral buoyancy transfer by baroclinic eddies and the buoyancy loss at the sea surface.

This localized forcing experiment is not appropriate, however, to investigate the effects of the preconditioning of the ocean on open-ocean deep convection since it assumes a horizontally homogeneous ocean as the initial condition. Furthermore, Straneo and Kawase (1999) pointed out that disk-cooling experiments are not equiv- 
alent to more realistic preconditioned experiments in which cooling is applied uniformly over the surface of the ocean preconditioned with a cyclonic gyre and the associated doming of isopycnals. In particular, they showed that the lateral density gradient decreases with time in preconditioned experiments contrary to diskcooling experiments.

Recently a few preconditioned experiments have been carried out to simulate open-ocean deep convection, in which the uniform surface cooling is applied on the ocean preconditioned by the domed isopycnals and the associated geostrophic velocity fields (Straneo and Kawase 1999; Legg et al. 1998; Legg and McWilliams 2000; Molemaker and Dijkstra 2000; Yoshikawa et al. 2001). In general, they found that baroclinic instability is generated from the initially stable cyclonic gyre under the influence of convection. Legg et al. (1998) also observed that a secondary circulation in the plane perpendicular to the original circulation is generated, thus causing the restratification of the convective column in the core of the gyre. Meanwhile, a density front and a downdraft along isopycnals are generated along with the generation of baroclinic eddies.

These experiments are concerned only with the cases in which the precondition always leads to baroclinic instability and the consequent localized deep convection. However, the generation of open-ocean deep convection accompanied by baroclinic instability may require a particular precondition. Especially, we can expect that if the initial flow structure cannot generate baroclinic instability soon after the start of convection, it becomes more difficult to generate it with the lapse of time since the radial buoyancy gradient decreases with time in preconditioned experiments, as pointed out by Straneo and Kawase (1999). This is contrary to the case of disk-cooling experiments where baroclinic instability always occurs with time as a result of the radial buoyancy gradient increasing continuously with time. Molemaker and Dijkstra (2000) also found that in preconditioned experiments the velocity of the rim current induced by convection is much smaller than that of the preconditioned cyclonic gyre, and thus the dominating unstable mode is determined by the preconditioned cyclonic gyre, not by the strength of the surface buoyancy flux.

Therefore we would like to understand how the preconditioning of the ocean affects the evolution of openocean deep convection in this paper. For this purpose, we carried out large-eddy simulations (LES) of openocean deep convection that is preconditioned by a cyclonic geostrophic gyre of various intensity and background stratification.

Meanwhile, we cannot rule out the possibility that the pattern of convection is affected by the magnitude of surface cooling as well as the preconditioning of the ocean. For example, Noh et al. (1999) showed that the generation of baroclinic eddies is suppressed in the diskcooling experiment if the surface buoyancy loss be- comes weaker. Yoshikawa et al. (2001) also suggested that convection plays a key role as an initiator of baroclinic instability in preconditioned experiments, although they did not examine the sensitivity on the magnitude of surface cooling. Therefore, we examined how the pattern of convection would be affected by the magnitude of the surface buoyancy flux as well.

Furthermore, we carried out dimensional analysis to find the appropriate nondimensional parameters that characterize the pattern of convection. The criterion, which determines the transition of the convection pattern, was obtained by analyzing the numerical results with different precondition and surface cooling.

\section{Model}

The LES model we used in this study is based on the nonhydrostatic Boussinesq equation. The model employs the periodic boundary condition at the sidewall and the stress-free boundary condition at the surface and the bottom. For a more detailed description of the model, see Raasch and Etling (1991).

Subgrid-scale turbulence is modeled according to Deardorff (1980). A prognostic equation is solved for the subgrid-scale turbulent kinetic energy (TKE) and the subgrid-scale eddy coefficients are related to the subgrid-scale TKE. The wide ranges of shear and stratification around the region of open-ocean deep convection are possibly taken into account by making use of the parameterization of the subgrid-scale turbulence. Large-eddy simulation of open-ocean deep convection has been attempted previously by Denbo and Skyllingstad (1996), Raasch and Etling (1998), and Noh et al. (1999).

The numerical scheme is a standard second-order finite-difference scheme using the absolutely conserving scheme of Piacsek and Williams (1970) for the nonlinear advection term. The prognostic equations are time-advanced by a leapfrog scheme. A weak time filter is applied to remove the time-splitting instability of the leapfrog scheme (Asselin 1972). During the integration the time step is adjusted so that it never exceeds onetenth of the allowed value because of the Courant-Friedrichs-Lewy (CFL) and diffusion criteria. Incompressibility is applied by means of the Poisson equation for pressure, which is solved by the FFT method. Recently the code has been parallelized, and the performance of the new parallelized code is found to be excellent on an SGI/Cray-T3E with an almost linear speed-up up to a very large number of processors (Raasch and Schröter 2001).

The initial condition is similar to Molemaker and Dijkstra (2000), although the values of parameters are different. The initial distribution of buoyancy $[b=-g(\rho$ $\left.-\rho_{0}\right) / \rho_{0}$, where $g, \rho$, and $\rho_{0}$ are the gravitational acceleration, the density, and a reference density, respectively] of the domed isopycnals in association with a cyclonic gyre is given by 
(a)
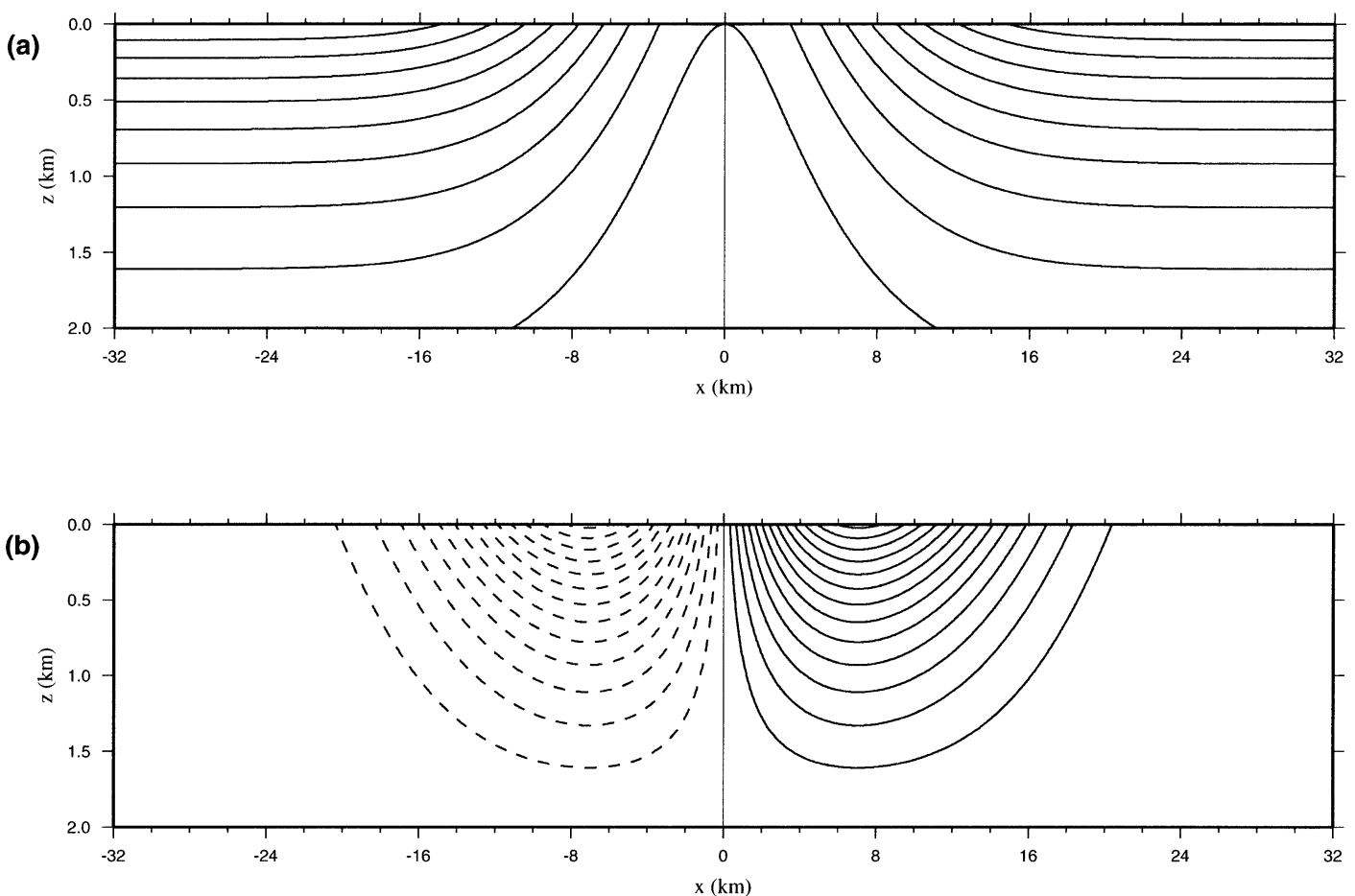

FIG. 1. The initial conditions of EXP A1. (a) The vertical cross section of buoyancy; range, $0-6.6 \times 10^{-4} \mathrm{~m} \mathrm{~s}^{-2}$; contour spacing, $0.7 \times 10^{-4} \mathrm{~m} \mathrm{~s}^{-2}$. (b) The vertical cross section of azimuthal velocity; range, $-0.44-0.44 \mathrm{~m} \mathrm{~s}^{-1}$; contour spacing, $0.04 \mathrm{~m} \mathrm{~s}^{-1}$, with positive values shown by solid contours and negative values by dotted contours.

$$
b=A e^{-\beta z}\left[1-\gamma e^{-(r / R)^{2}}\right],
$$

where $R$ and $\beta$ represent the horizontal and vertical scales of the gyre, respectively. Here $r$ is the distance from the center of the gyre, and $z$ is the downward distance from the surface. The intensity of the gyre is controlled by $A$ and $\gamma$, and the background stratification away from the gyre is given by $N^{2}=A \beta e^{-\beta z}$. The corresponding azimuthal velocity of the gyre $u_{\theta}$, which is in geostrophic balance with the buoyancy field, is given by

$$
u_{\theta}=2 \frac{A \gamma}{f \beta R^{2}} r e^{-(r / R)^{2}}\left(e^{-\beta z}-e^{-\beta H}\right),
$$

with the neglect of the cyclostrophic term $u_{\theta}^{2} / r$, where $f$ is the Coriolis parameter and $H$ is the vertical scale of the domain. Note that the velocity field (2) disappears at $z=H$. An example of the cross section of the initial buoyancy and velocity field is shown in Fig. 1. The maximum values of the radial buoyancy gradient and the azimuthal velocity, both of which represent the intensity of the cyclonic gyre, appear at $r=R^{*}(=R / \sqrt{2})$ and are proportional to $A \gamma$. We will call $r=R^{*}$ the rim of a cyclonic gyre.

The simulation requires a sufficiently fine grid spacing to resolve the small-scale convective plumes and a sufficiently large horizontal domain to include the evolution of baroclinic instability around the gyre. Accordingly we employed the computational domain as 64 $\mathrm{km} \times 64 \mathrm{~km} \times 2 \mathrm{~km}$ with a grid size of $250 \mathrm{~m} \times 250$ $\mathrm{m} \times 100 \mathrm{~m}$, which requires $256 \times 256 \times 20$ grid points. Throughout the experiments the precondition was varied by changing $A$ and $\gamma$. The ranges of parameters in the experiments were $A=0.3-6.6\left(\times 10^{-4} \mathrm{~m} \mathrm{~s}^{-2}\right)$ and $\gamma$ $=0.1-0.9$ (see Table 1$)$. Other parameters were fixed as $R=10^{4} \mathrm{~m}$ (or $\left.R^{*} \cong 7.1 \times 10^{3} \mathrm{~m}\right), \beta=10^{-3} \mathrm{~m}^{-1}$, and $f=10^{-4} \mathrm{~s}^{-1}$. This gives the range of stratification at the surface as $N_{0}^{2}=0.3-6.6 \times 10^{-7} \mathrm{~m} \mathrm{~s}^{-2}$, which roughly represents the stratification at the sites of openocean deep convection.

The surface buoyancy loss $B$ was given by $B=10^{-7}$ $\mathrm{m}^{2} \mathrm{~s}^{-3}$ as the typical value, but for the series of experiments investigating the effects of the surface cooling, a wide range of $B$ was applied $\left(B=10^{-9}-10^{-6} \mathrm{~m}^{2} \mathrm{~s}^{-3}\right.$; see Table 1).

The integration was carried out over a period of 8 days. Cooling events to generate open-ocean deep convection last only a few days typically (Marshall and Schott 1999). We presume the stability of the initial condition, since the simulation without the surface cooling in the case of the strongest baroclinity, or the largest $u_{\theta}^{*}$, is found to be stable to baroclinic instability during this period.

\section{Dimensional analysis}

Molemaker and Dijkstra (2000) and Legg et al. (1998) showed that a cyclonic gyre with the stable initial con- 
(a)
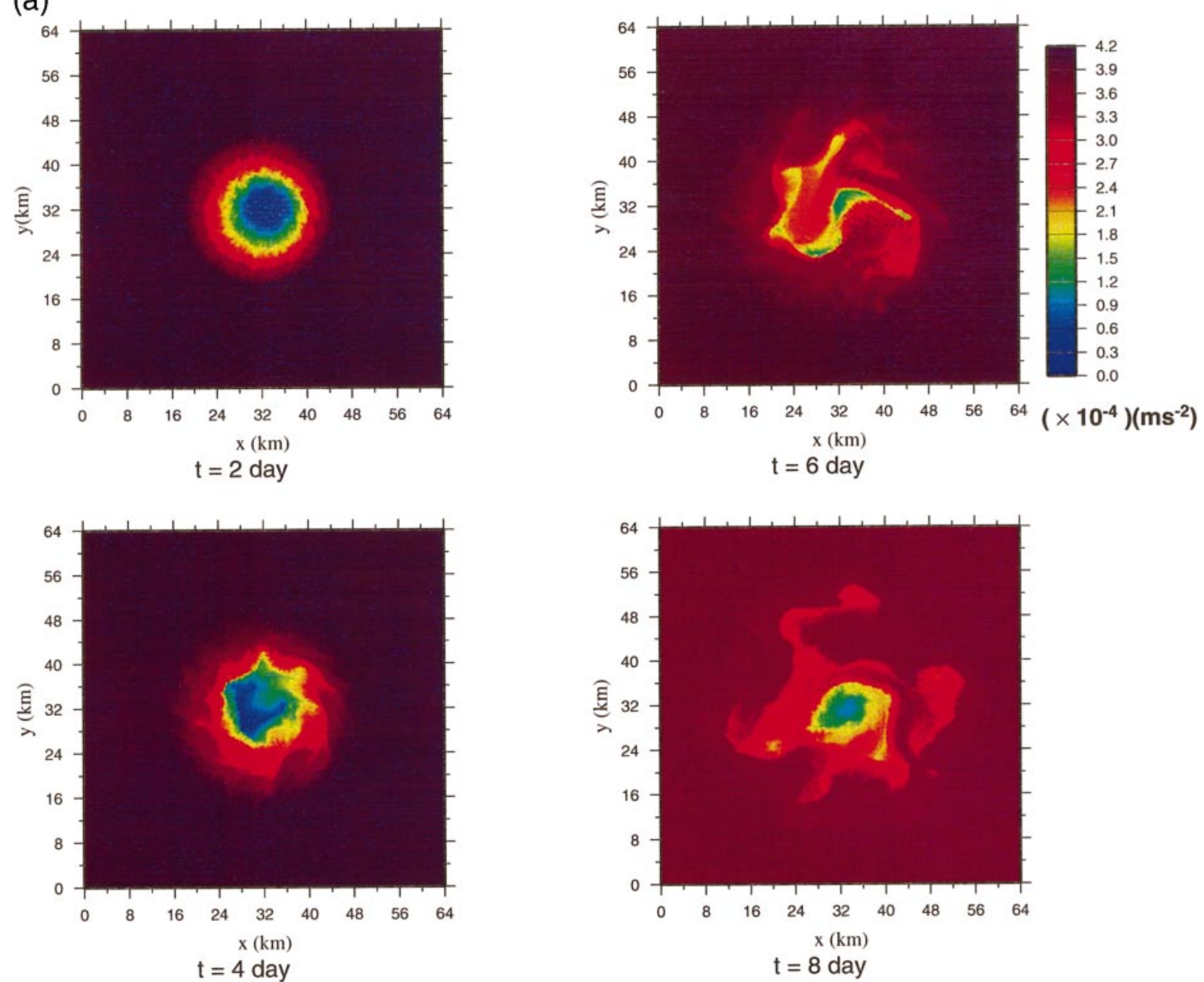

FIG. 2. Buoyancy distribution at the surface from EXP A: (a) EXP A1 $\left(A=6.67 \times 10^{-4} \mathrm{~m} \mathrm{~s}^{-2}\right)(t=2,4,6,8$ days); (b) EXP A4 (A= $\left.0.33 \times 10^{-4} \mathrm{~m} \mathrm{~s}^{-2}\right)(t=4,8$ days). Note that the figures are shown less frequently for EXP A4 because the pattern remains similar.

figuration becomes baroclinically unstable under convection, as $\tilde{L}_{V} / R$ becomes smaller along with the erosion of the stratification in the upper layer, where $\tilde{L}_{V}$ is the Rossby radius of deformation in terms of vertical density gradient such as

$$
\tilde{L}_{V}=N H / f,
$$

and $H$ is the vertical length scale. It is also in agreement with the theoretical prediction of baroclinic instability of a two-layer quasigeostrophic vortex (Pedlosky 1985).

Meanwhile, Molemaker and Dijkstra (2000) also showed that a cyclonic gyre becomes increasingly susceptible to baroclinic instability with increasing $\tilde{L}_{H} / R$, where $\tilde{L}_{H}$ is the Rossby radius of deformation in terms of the horizontal density gradient. Here we can define $\tilde{L}_{H}$ in terms of the buoyancy difference between the core of a cyclonic gyre and the outer region $\Delta b$, that is,

$$
\tilde{L}_{H}=\frac{\sqrt{\Delta b H}}{f} .
$$

The parameter $\tilde{L}_{H} / R$ has been also used to predict the generation of baroclinic instability in laboratory experiments in which a rotating annulus of fluid is subjected to radial buoyancy gradient or heating (Hide 1958; Fowlis and Hide 1965; Saunders 1973; Griffiths and Linden 1981; Tritton and Davies 1985).

In the case of disk-cooling experiments, $H$ is determined by the depth of the convective boundary layer $h$ below the disk cooling, and the buoyancy anomaly of the convective region from the background is obtained as

$$
\Delta b \sim N^{2} h,
$$

if the initial stratification is given by constant stratification $N^{2}$. Here the growth of $h$ is often predicted by 
(b)

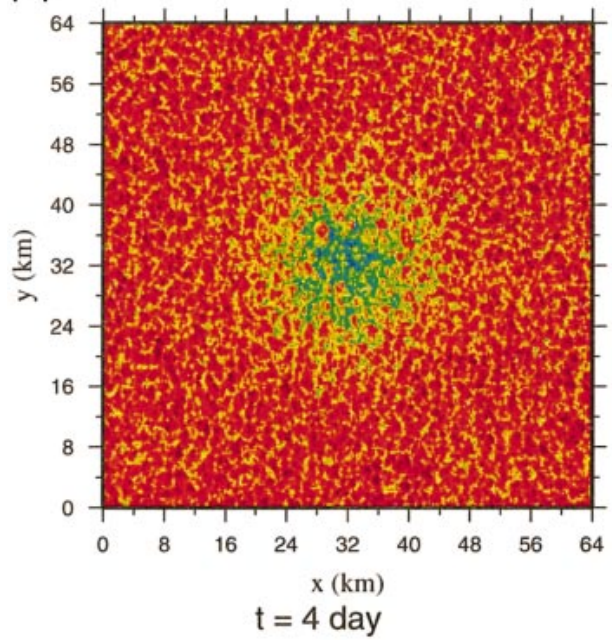

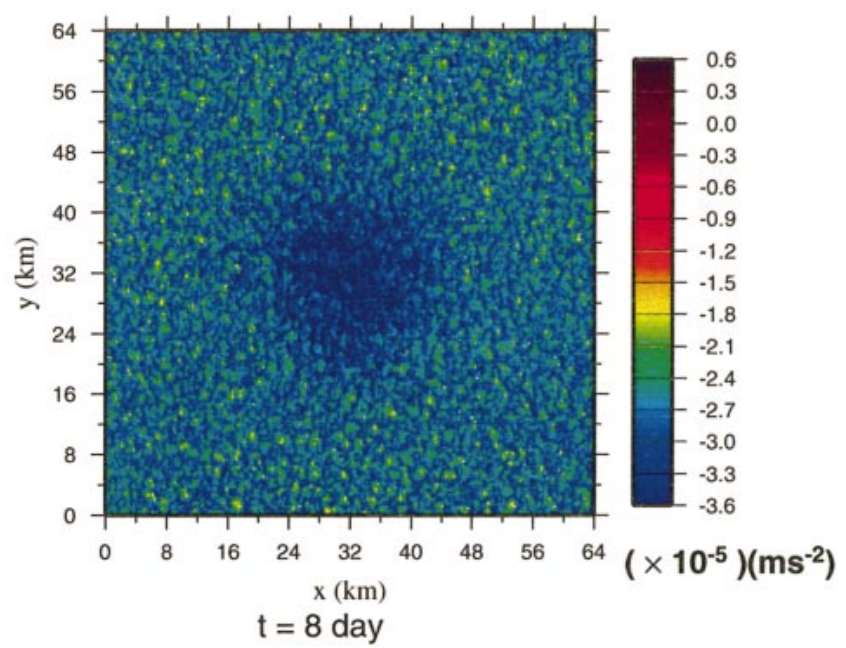

FIG. 2. (Continued)

assuming the homogeneous convective boundary layer without entrainment at its base as (Turner 1973)

$$
h=\frac{(2 B t)^{1 / 2}}{N}
$$

when $N$ is constant.

Note that in disk-cooling experiments, according to (5) and (6), $\tilde{L}_{H} / R$ always increases with time as $h$ in-

TABLE 1. The precondition and the surface buoyancy flux for all the simulations together with the corresponding parameters. The last column represents the resultant pattern of convection; open circles: localized convection; crosses: distribution convection; open triangles: the intermediate pattern.

\begin{tabular}{lccccccc}
\hline \hline & $A \times 10^{4}$ & $\gamma$ & $B \times 10^{7}$ & Ro & Bu & Ta $\times 10^{-6}$ & \\
\hline A1 & 6.67 & 0.9 & 1.0 & 0.60 & 0.82 & 0.44 & $\bigcirc$ \\
A2 & 1.67 & 0.9 & 1.0 & 0.15 & 0.41 & 0.028 & $\bigcirc$ \\
A3 & 0.67 & 0.9 & 1.0 & 0.06 & 0.26 & 0.0045 & $\times$ \\
A4 & 0.33 & 0.9 & 1.0 & 0.03 & 0.18 & 0.0011 & $\times$ \\
B1 & 1.67 & 0.9 & 1.0 & 0.15 & 0.41 & 0.028 & $\bigcirc$ \\
B2 & 1.67 & 0.6 & 1.0 & 0.10 & 0.41 & 0.028 & $\bigcirc$ \\
B3 & 1.67 & 0.3 & 1.0 & 0.05 & 0.41 & 0.028 & $\triangle$ \\
B4 & 1.67 & 0.1 & 1.0 & 0.02 & 0.41 & 0.028 & $\times$ \\
C1 & 6.67 & 0.9 & 0.25 & 0.60 & 0.82 & 7.12 & $\bigcirc$ \\
C2 & 1.67 & 0.9 & 0.25 & 0.15 & 0.41 & 0.45 & $\bigcirc$ \\
C3 & 0.67 & 0.9 & 0.25 & 0.06 & 0.26 & 0.072 & $\triangle$ \\
C4 & 0.33 & 0.9 & 0.25 & 0.03 & 0.18 & 0.017 & $\times$ \\
D1 & 1.67 & 0.9 & 0.01 & 0.15 & 0.41 & 278.9 & $\bigcirc$ \\
D2 & 1.67 & 0.9 & 0.25 & 0.15 & 0.41 & 0.45 & $\bigcirc$ \\
D3 & 1.67 & 0.9 & 1.0 & 0.15 & 0.41 & 0.028 & $\bigcirc$ \\
D4 & 1.67 & 0.9 & 2.5 & 0.15 & 0.41 & 0.0045 & $\triangle$ \\
D5 & 1.67 & 0.9 & 10.0 & 0.15 & 0.41 & 0.0003 & $\times$ \\
E1 & 6.67 & 0.1 & 1.0 & 0.07 & 0.82 & 0.44 & $\bigcirc$ \\
E2 & 6.67 & 0.1 & 10.0 & 0.07 & 0.82 & 0.0044 & $\times$ \\
F1 & 1.67 & 0.9 & 1.0 & 0.15 & 0.41 & 0.028 & $\bigcirc$ \\
F2 & 15.03 & 0.1 & 9.0 & 0.15 & 1.23 & 0.028 & $\bigcirc$ \\
F3 & 5.01 & 0.3 & 3.0 & 0.15 & 0.71 & 0.028 & $\bigcirc$ \\
G1 & 1.67 & 0.1 & 1.0 & 0.02 & 0.41 & 0.028 & $\times$ \\
G2 & 0.185 & 0.9 & 0.11 & 0.02 & 0.14 & 0.028 & $\times$ \\
\hline
\end{tabular}

creases under convection, while $\tilde{L}_{\mathrm{V}} / R$ decreases owing to the erosion of stratification. This implies that baroclinic instability always occurs after sufficient time in this case.

In preconditioned experiments, however, $\tilde{L}_{H}$ decreases with time, since $\Delta b$ decreases with increasing $h$ under convection, owing to the domic structure of isopycnals such as (1) (Straneo and Kawase 1999). This suggests that baroclinic instability may not occur under a certain condition despite the erosion of stratification under convection.

The relevant variables that appear in the present simulations are $A, \beta, \gamma, f, R$, and $B$. Here the intensity of a gyre $\gamma$ can be replaced by the azimuthal velocity scale of a cyclonic gyre $u_{\theta}^{*}$ as

$$
u_{\theta}^{*}=A \gamma / f \beta R .
$$

We can also replace $A$ and $\beta$ by $N_{0}=(A \beta)^{1 / 2}$ and $H=$ $\beta^{-1}$, where $N_{0}$ represents the initial background stratification at the surface.

It is then possible to assume that the pattern of convection, such as the occurrence of baroclinic instability, is determined by

$$
F\left(u_{\theta}^{*}, B, N_{0}, H, f, R\right)=0 .
$$

Dimensional analysis of (8) in terms of $f$ and $R$ leads to

$$
\begin{aligned}
& F\left(\frac{u_{\theta}^{*}}{f R}, \frac{B}{f^{3} R^{2}}, \frac{N_{0}}{f}, \frac{H}{R}\right)=0 \quad \text { or } \\
& F\left(\frac{L_{H}}{R}, \frac{L_{V}}{R}, \frac{B}{f^{3} R^{2}}, \frac{H}{R}\right)=0,
\end{aligned}
$$

where $\left(L_{H} / R\right)^{2}=u_{\theta}^{*} / f R$ and $L_{V} / R=N_{0} H / f R$ represent the initial values of $\tilde{L}_{H} / R$ and $\tilde{L}_{V} / R$. Here $\left(L_{H} / R\right)^{2}$ corresponds to the Rossby number Ro associated with a cyclonic gyre, and $L_{V} / R$ corresponds to the Burger num- 

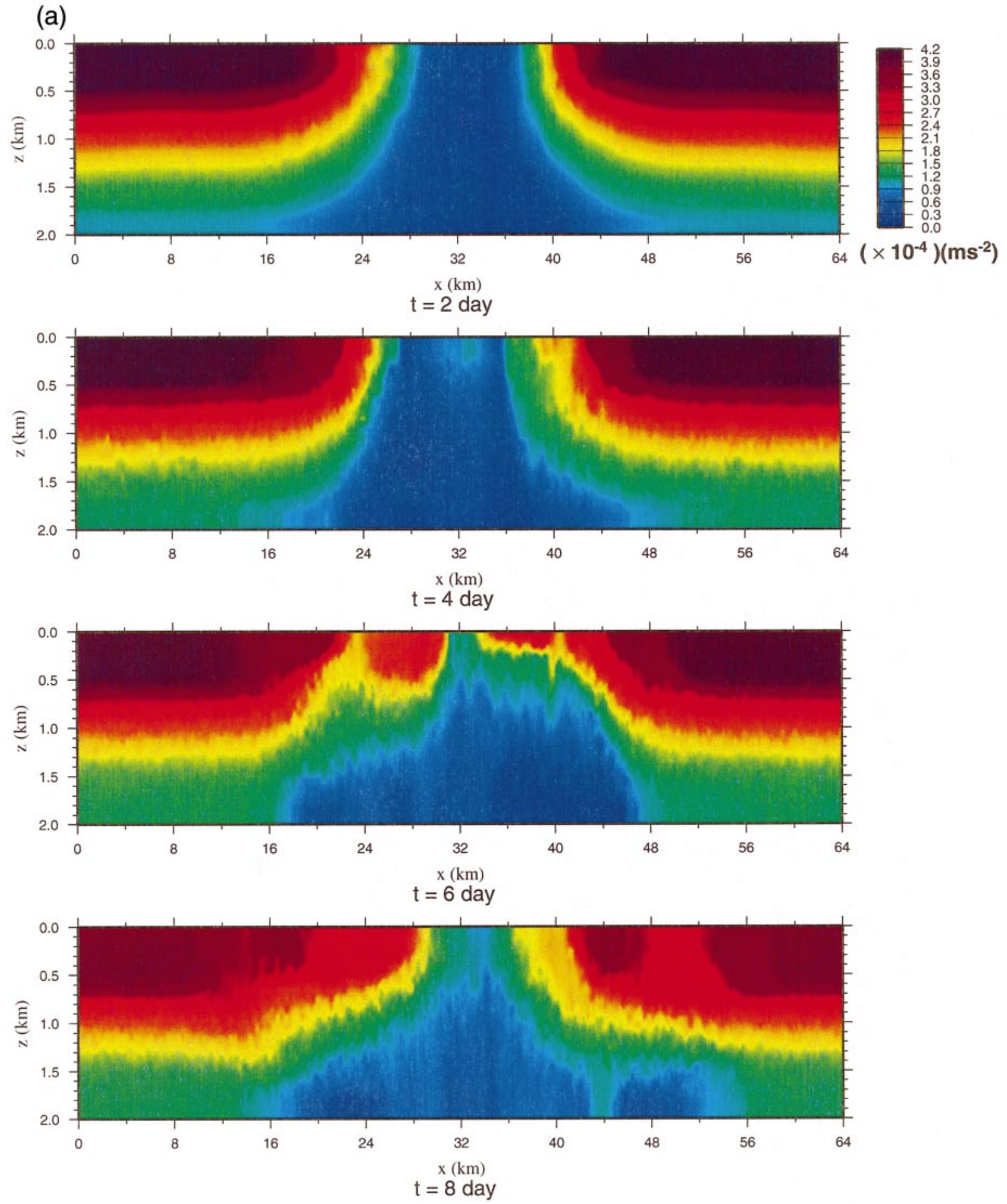

FIG. 3. Buoyancy distribution at the vertical cross section from EXP A: (a) EXP A1 $\left(A=6.67 \times 10^{-4} \mathrm{~m} \mathrm{~s}^{-2}\right)(t=2,4,6,8$ days); (b) EXP A4 $\left(A=0.33 \times 10^{-4} \mathrm{~m} \mathrm{~s}^{-2}\right)(t=4,8$ days $)$. 
(b)
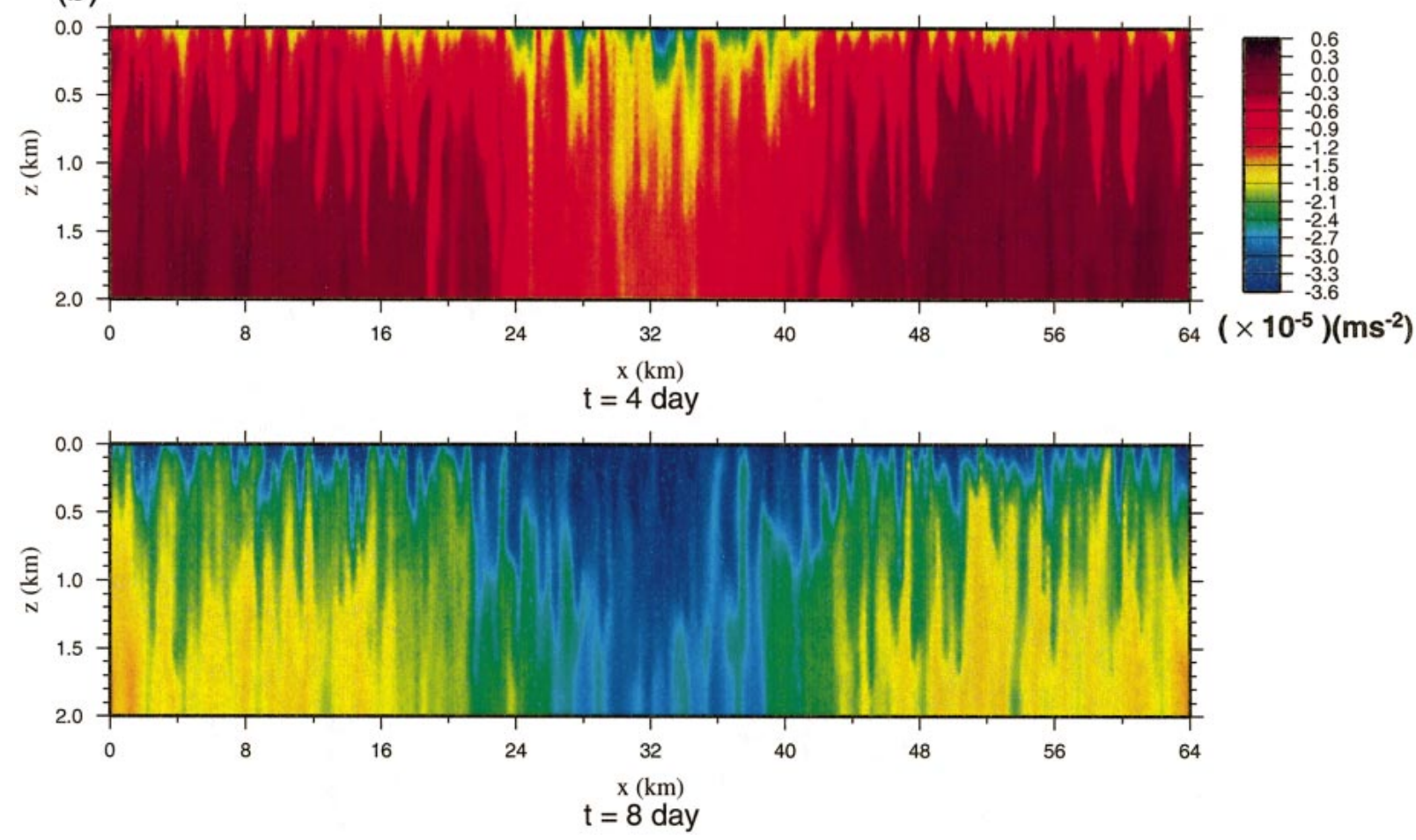

FIG. 3. (Continued)

ber $\mathrm{Bu}$. We can regard $B / f^{3} R^{2}$ as another Rossby number associated with convection.

In the present simulations, Ro and Bu can be scaled as

$$
\begin{aligned}
& \mathrm{Ro} \sim\left(\frac{L_{H}}{R}\right)^{2} \sim \frac{A \gamma / \beta}{f^{2} R^{2}} \text { and } \\
& \mathrm{Bu} \sim \frac{L_{V}}{R} \sim \frac{N_{0} H}{f R} \sim \frac{(A / \beta)^{1 / 2}}{f R} .
\end{aligned}
$$

We can expect that the surface buoyancy loss affects the flow primarily by eroding stratification and increasing the TKE level, both of which are also controlled by the background stratification. Hence it may be more appropriate to represent the effects of $B$ in combination with $N_{0}$. From this perspective, we can replace $B / f^{3} R^{2}$ by

$$
\frac{B}{f^{3} R^{2}}\left(\frac{N_{0}}{f}\right)^{-2} \sim \frac{B / N_{0}^{2}}{f R^{2}} .
$$

If we can estimate the eddy diffusivity $\kappa_{T}$ generated from the convection imposed on the stratification $N_{0}$ by $B \sim \kappa_{T} N_{0}^{2}$, we can rewrite (13) as

$$
\frac{B / N_{0}^{2}}{f R^{2}}\left(\frac{R}{H}\right)^{2} \sim \frac{\kappa_{T}}{f H^{2}} \sim \mathrm{Ta}^{-1 / 2},
$$

where Ta is the Taylor number based on the eddy viscosity.
Consequently, we can rewrite (10) as

$$
F(\mathrm{Ro}, \mathrm{Bu}, \mathrm{Ta}, H / R)=0,
$$

by using the definitions as $\mathrm{Ro}=u_{\theta}^{*} / f R, \mathrm{Bu}=N_{0} H / f R$, and $\mathrm{Ta}=\left(B / N_{0}^{2} f H^{2}\right)^{-2}$. In the present experiments, $H / R$ remains invariant.

\section{Numerical experiments}

We carried out several series of experiments with the purpose of investigating how the characteristics of convection are affected by the parameters appearing in (15). Variables of each experiment and the corresponding parameters in (15) are listed in Table 1.

First, we performed two series of experiments; one with only $A$ varied (EXP A) and the other with only $\gamma$ varied (EXP B). This means that only Ro varies during EXP B, but all three parameters Ro, Bu, and Ta vary simultaneously during EXP A. EXP C is the same as EXP A except that the surface buoyancy loss $B$ is smaller. Meanwhile, during EXP D and EXP E, the effects of $B$ are investigated, in which Ta is the only varying parameter. Last, we investigated the effects of $\mathrm{Bu}$ by carrying out series of experiment in which $\mathrm{Bu}$ is changed while Ro and Ta are fixed (EXP F and EXP $\mathrm{G) \text {. }}$

For an individual experiment belonging to a series of experiments mentioned above, we named it by attaching 
(a)
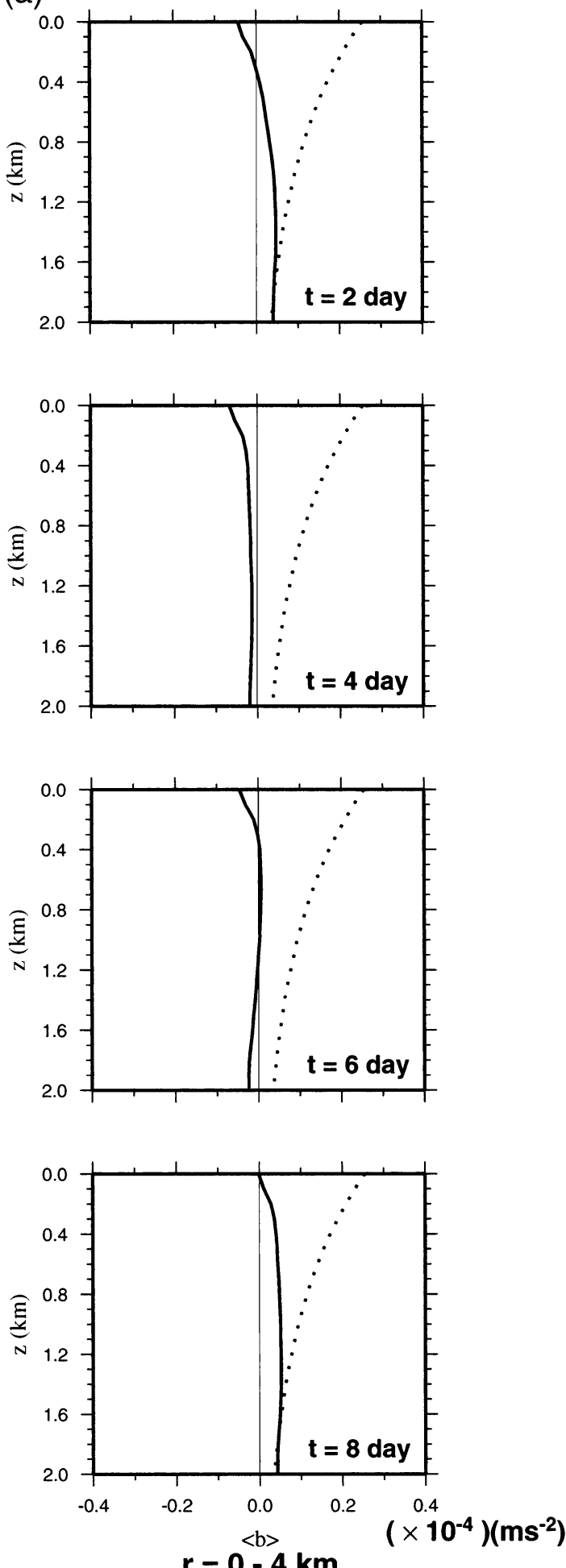
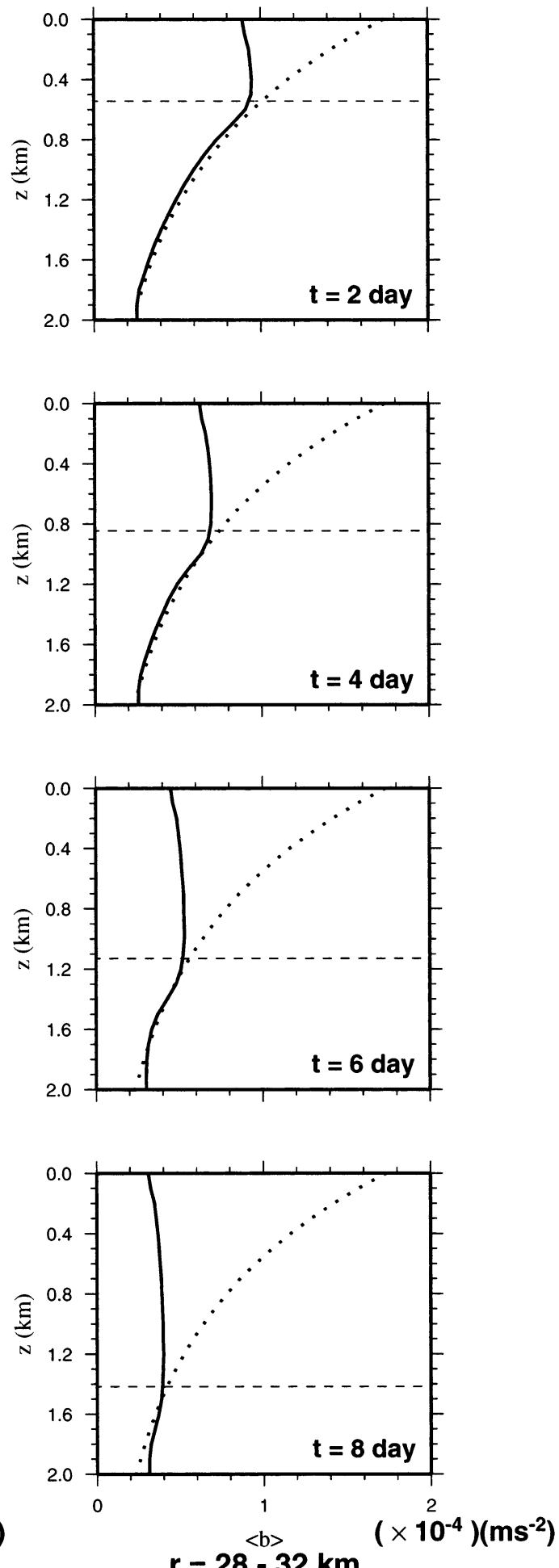

FIG. 4. Vertical profiles of the azimuthally averaged buoyancy over the radial thickness of $4 \mathrm{~km}$ in the core of the gyre $(r=0-4 \mathrm{~km})$ and in the exterior region $(r=28-32 \mathrm{~km})$ at $t=2,4,6,8$ days. Solid: simulation results; dotted: the initial profile. The predicted depths of the convective boundary layer $h$ from (7) are shown by a dashed line for (a) EXP B1 and (b) EXP B4. (In the profile at $r=0-4 \mathrm{~km}$ of EXP B1, $h$ is larger than $2 \mathrm{~km}$ from day 2.) 
(b)
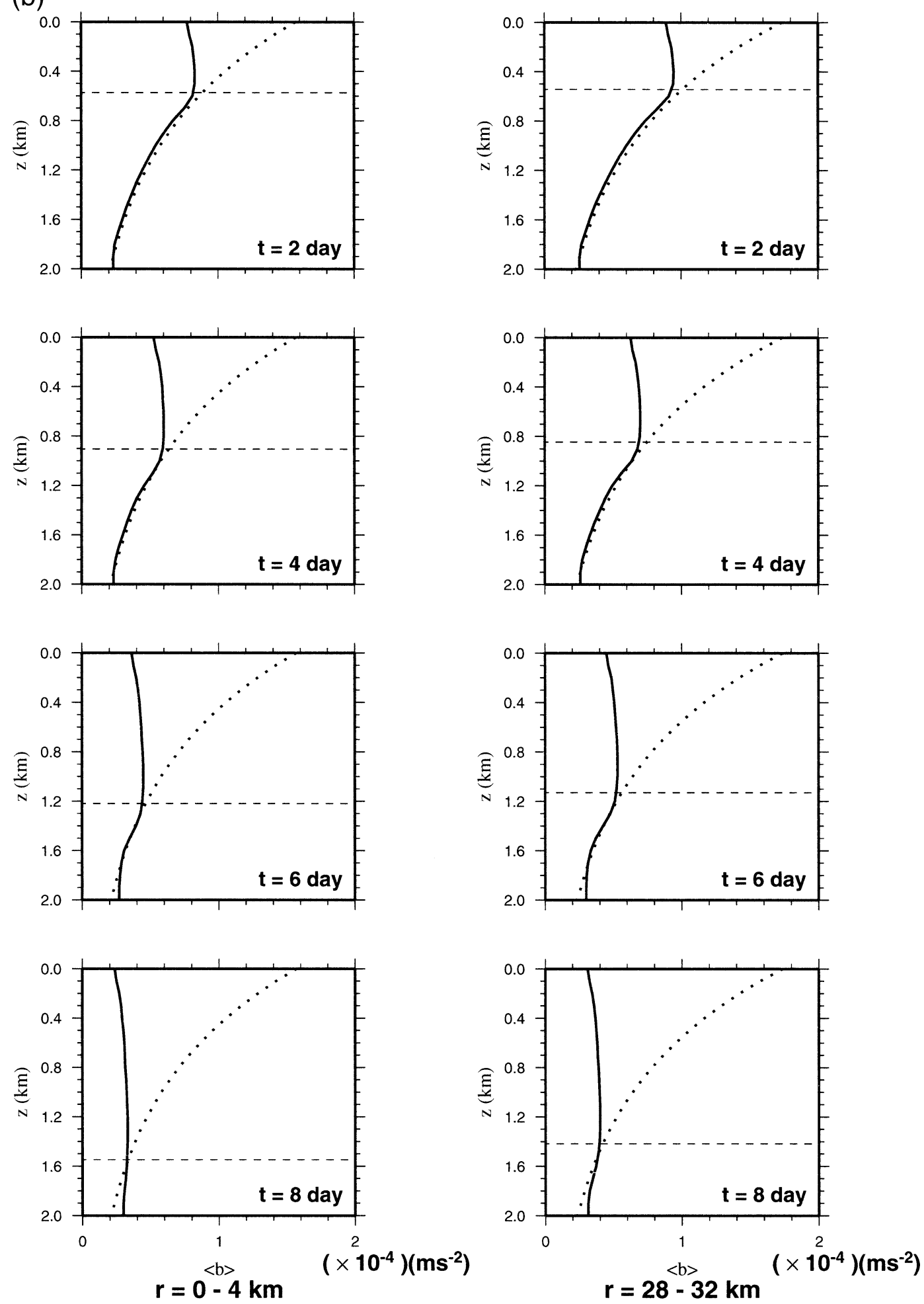

FIG. 4. (Continued) 
a number following the name of a series of experiments. For example, EXP A2 is the second experiment of EXP A. In many cases the same experiment belongs to different series of experiments. For example, EXP A2, EXP B1, EXP D3, and EXP F1 are an identical experiment. The same is true for EXP C2 and EXP D2 and for EXP B4 and EXP G1.

\section{Results}

a. Variation of convection pattern according to the precondition

Figures 2 and 3 show the vertical and horizontal cross sections of buoyancy field at various stages of the evolution of $\operatorname{EXP} \mathrm{A}\left(A=0.33,6.6 \times 10^{-4} \mathrm{~m} \mathrm{~s}^{-2}, \gamma=\right.$ $0.9)$.

When $A$ is large $\left(\mathrm{EXP} \mathrm{A} 1 ; A=6.6 \times 10^{-5} \mathrm{~m} \mathrm{~s}^{-2}\right.$; Figs. $2 \mathrm{a}$ and $3 \mathrm{a}$ ), the condition and the surface buoyancy flux of this experiment are very similar to the reference experiment of Legg et al. (1998), although constant eddy viscosity and diffusivity were used in the latter. Accordingly, the very similar evolution of convection appears.

Initially, small-scale convective plumes appear over the whole surface, although they are stronger in the region of weaker stratification at the core of the gyre, which is clearly manifested in the horizontal distribution of vertical velocity (not shown; see, e.g., Legg et al. 1998; Molemaker and Dijkstra 2000). Strong mixing within the core of the gyre merges convective plumes to form a convective column in the center of the radius of about $R^{*}$, which is separated from the exterior by a front. Outside the convective column the small-scale convective plumes still persist. Baroclinic instability appears soon after the start of surface cooling (after 1 day) (see also Fig. 10), and small-scale meandering develops along the rim of the gyre $\left(r \sim R^{*}\right)$. The vertical velocity field also shows a very similar structure to those in Legg et al. (1998) and Molemaker and Dijkstra (2000) with the swirling pattern in the core of the gyre (not shown).

The restratification in the convective column is also observed after some time (Fig. 3a), as in the case of Legg et al. (1998). It starts roughly at the same time as the generation of baroclinic instability. At the later stage the meandering grows into eddies that are comparable in size to the radius of the cyclonic gyre $R^{*}$. Subsequently baroclinic eddies migrate away from the gyre (day 8 of Fig. 2a; see also Fig. 7a). The migration of baroclinic eddies and the subsequent breakup of the cyclonic gyre severely modify the vertical structure of buoyancy as shown in day 8 of Fig. 3a.

In the exterior region the buoyancy of the upper part continues to decrease with time. In the interior region, however, the buoyancy starts to increase over the whole depth owing to strong lateral mixing by baroclinic eddies and secondary circulation (see also Fig. 4a). This trend is enhanced further with the breakup of the cy- clonic gyre. Therefore the radial buoyancy gradient decreases significantly with time.

On the other hand, when $A$ is much smaller (EXP A4; $A=0.3 \times 10^{-5} \mathrm{~m} \mathrm{~s}^{-2}$; Figs. $2 \mathrm{a}$ and $3 \mathrm{~b}$ ), smallscale convective plumes appear uniformly over the whole surface, similarly to the convective boundary layer, without forming a core of violent mixing at the center of the gyre. The vertical velocity fluctuates almost uniformly over the whole surface (not shown). Neither baroclinic instability nor restratification appears in this case. In a certain sense the progress of convection resembles the two-dimensional convection in which baroclinic instability cannot be induced (Yoshikawa et al. 2001).

Similar contrast is also observed from EXP B (not shown). Here the growth of baroclinic eddies are slower in EXP B1 (or EXP A2) than in EXP A1, as expected from the linear stability theory by Eady (1949) for the growth rate $\sigma$ of the disturbance as

$$
\sigma \sim \frac{f}{N} \frac{\partial u_{\theta}}{\partial z} \sim \frac{A^{1 / 2} \gamma}{\beta^{1 / 2} R} .
$$

It is also observed that the convective patterns in the exterior region are the same in EXP B1 and EXP B4.

To distinguish two fundamentally different patterns of convection we will call the former "localized convection" (EXP A1 and EXP B1) and the latter "distributed convection", (EXP A4 and EXP B4) hereinafter.

The contrast between the two different convection processes is also clearly shown in the evolution of vertical buoyancy profiles for the case of EXP B (Fig. 4). Noticeably the buoyancy within the core is found to decrease at first and then to increase afterward along with the appearances of restratification and baroclinic eddies in EXP B1, whereas it decreases continuously in the exterior region. It is a contrast to the case of EXP B4 where the buoyancy continues to decrease with time both in the core of the gyre and in the exterior region. We also observed that the actual depth of the convective boundary layer in the exterior region is in good agreement with the prediction by (6), as observed by Ivey et al. (1995) and Raasch and Etling (1998). The equivalent contrast is also found in the vertical profiles of buoyancy from EXP A.

\section{b. Evolutions of radial distributions of variables}

In this section we investigate the evolution of the radial distribution of various azimuthally averaged variables for the experiments shown above, taking into consideration the contrast in the pattern of convection described above. Here $\bar{X}$, or $\langle X\rangle$, represents the azimuthal average of a variable $A$ with respect to the origin at the center of a cyclonic gyre, and $X^{\prime}$ represents the fluctuating component of $X$; that is, $X^{\prime}=X-\bar{X}$.

The most conspicuous distinction between the two types of convection appears in the radial distribution of horizontal TKE $\left[E_{h}=\left(\overline{u_{r}^{\prime 2}}+\overline{u_{\theta}^{\prime 2}}\right) / 2\right]$ of the surface (Fig. 
(a)

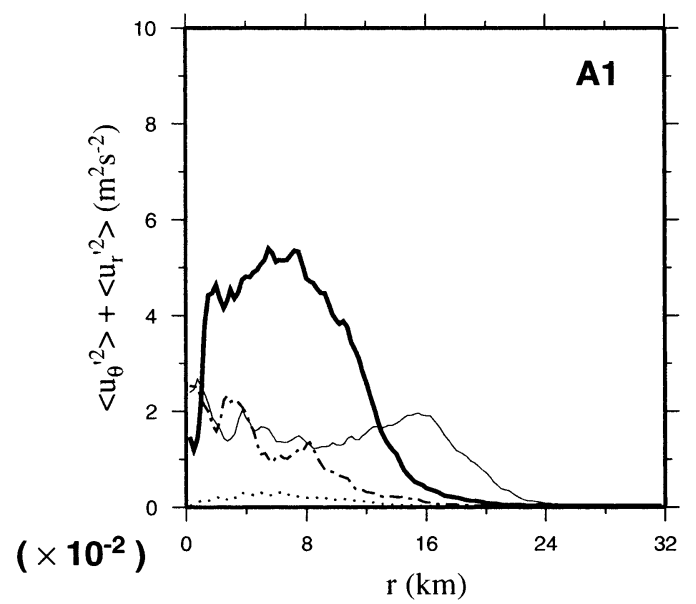

(b)

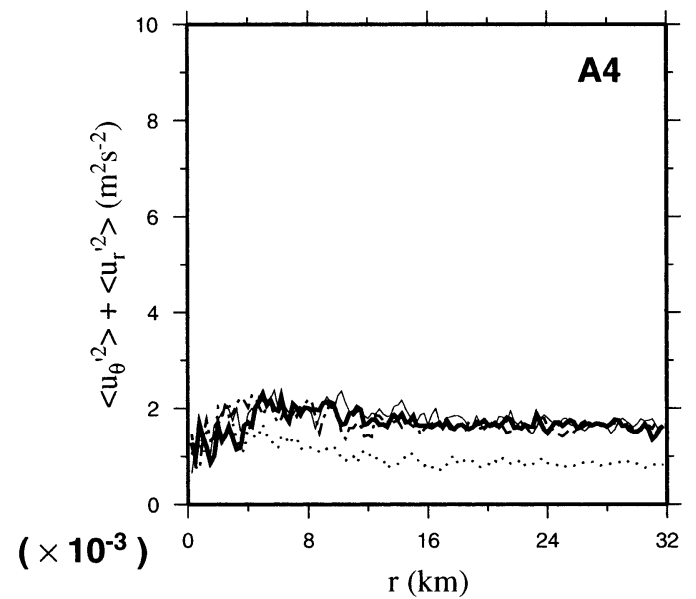

(c)

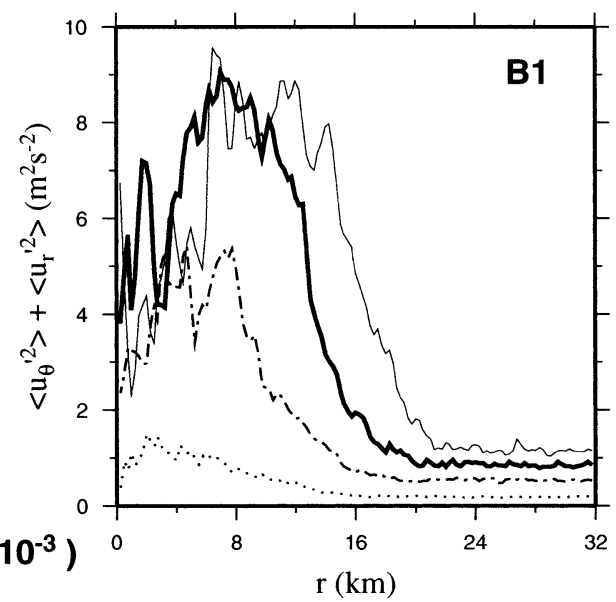

(d)

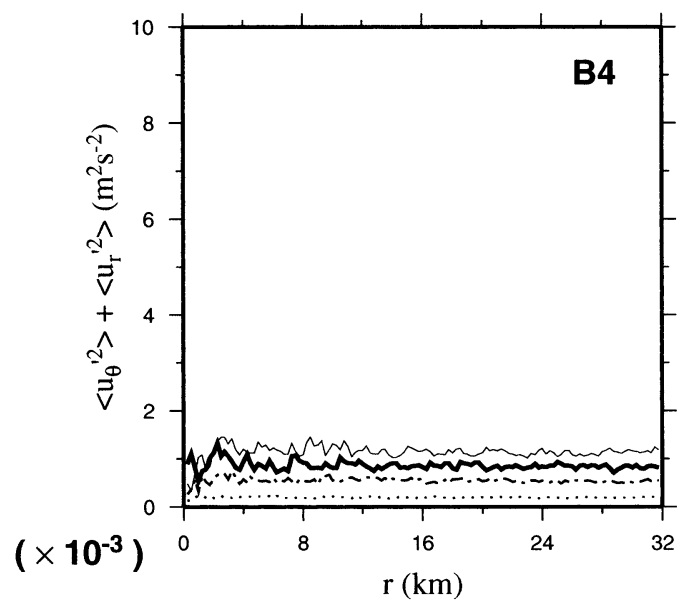

FIG. 5. Evolution of the radial distribution of the azimuthally averaged horizontal turbulent kinetic energy $E_{h}$ $\left[=\left(\overline{u_{r}^{\prime 2}}+\overline{u_{\theta}^{\prime 2}}\right) / 2\right]$ at the surface (dotted: day 2; dot-dashed: day 4; thick solid: day 6; thin solid: day 8) for (a) EXP A1, (b) EXP A4, (c) EXP B1, and (d) EXP B4. [Note that the vertical scale of Fig. 5a (EXP A1) is 10 times as large.]

5). Here we neglect the subgrid-scale TKE that is much smaller than the resolved TKE. In localized convection (EXP A1 and EXP B1) the strong peak of $E_{h}$ appears at $r \sim R^{*}(\cong 7.1 \mathrm{~km})$, owing to the generation of baroclinic eddies, and it keeps increasing with time. However, after the breakup of the convective column, $E_{h}$ spreads over much larger radial distances and its peak intensity decreases (day 8 of EXP A1). On the other hand, in distributed convection (EXP A4 and EXP B4), $E_{h}$ remains almost uniform over the whole region with the magnitude an order smaller than that of EXP A1. In particular, the difference in $E_{h}$ at the peak and in the background region does not increase with time contrary to the case of localized convection. This will be used in the next section to distinguish between localized convection and distributed convection.

Figure 6 shows the evolution of the vertical TKE distributions $\left(E_{v}=\overline{w^{\prime 2}} / 2\right)$ near the surface $(z=100$ $\mathrm{m})$. It generally reproduces the results expected from the convective boundary layer of the ocean (i.e., Denbo and Skyllingstad 1996). In localized convection (EXP A1 and EXP B1), initially $E_{v}$ is larger in the core of the gyre, reflecting the weaker stratification there. However, it is suppressed after day 4 , as the restratification starts. On the other hand, in distributed convection (EXP A4 and EXP B4) the distribution of $E_{v}$ is uniform after the initial stage because either $h$ reaches to the bottom (EXP A4) or it does not vary much in the initial configuration (EXP B4).

The inducement of the secondary circulation, which causes the restratification in the core of the gyre, is manifested in the evolution of the radial velocity at the surface $\bar{u}_{r}$ (Fig. 7). In localized convection, $\bar{u}_{r}$ is induced toward the center of the gyre initially, as shown in EXP B1 and the initial stage of EXP A1 (day 2). If baroclinic eddies become stronger, however, the secondary circulation converges toward the rim of the convective column $\left(r \sim R^{*}\right)$ (day 6 of EXP A1). A complex pattern, 
(a)

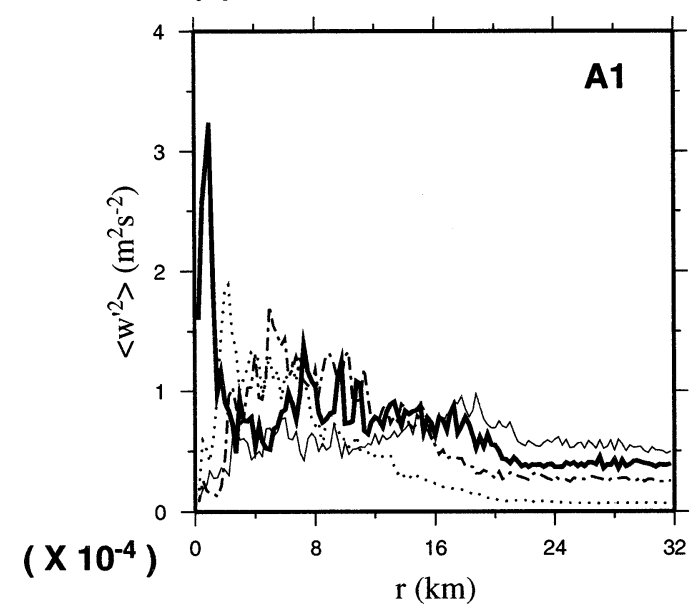

(b)

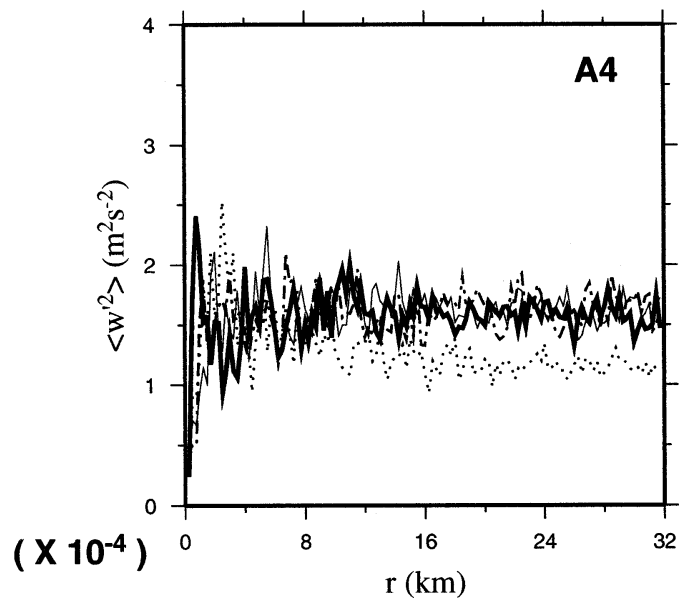

(c)

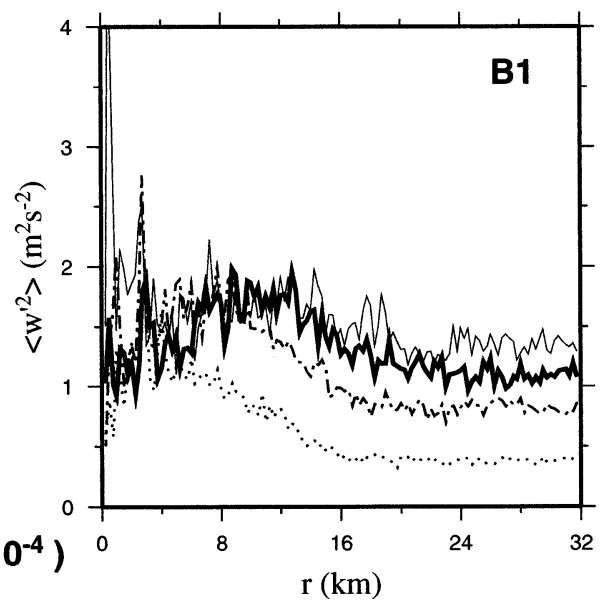

(d)

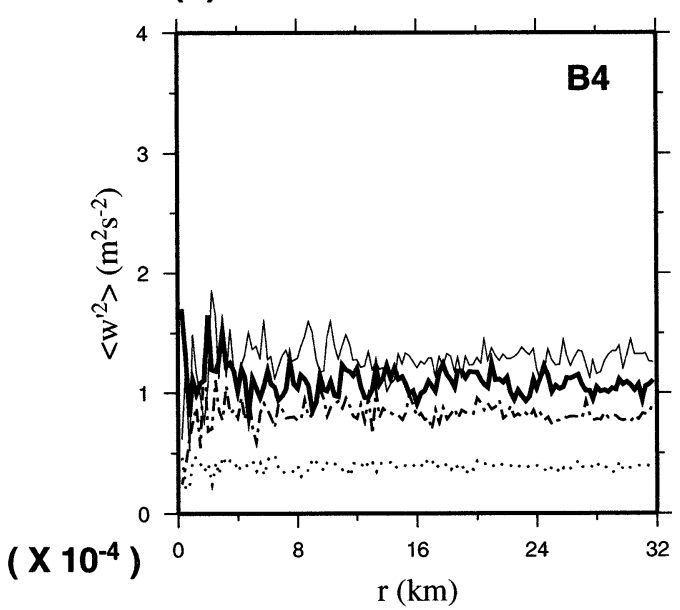

FIG. 6. Evolution of the radial distribution of the azimuthally averaged vertical turbulent kinetic energy $E_{v}\left(=\overline{w^{\prime 2}} / 2\right)$ at $z=100 \mathrm{~m}$ (line patterns correspond to days as in Fig. 5) for (a) EXP A1, (b) EXP A4, (c) EXP B1, and (d) EXP B4.

which spreads over larger radial distance, appears at later time as baroclinic eddies migrate away from the gyre (day 8 of EXP A1). The general pattern is also in agreement with Legg et al. (1998) and Yoshikawa et al. (2001). Meanwhile, in distributed convection no noticeable secondary circulation appears (EXP A4 and EXP B4).

The contrast between the two convection regimes is also found in the evolution of the mean azimuthal velocity $\bar{u}_{\theta}$ (Fig. 8). The distribution of $\bar{u}_{\theta}$, which remains unaffected until the onset of baroclinic instability, is modified significantly with the further progress of baroclinic instability in localized convection (after day 6 of both EXP A1 and EXP B1). It may be caused by the lateral mixing of angular momentum by baroclinic eddies and secondary circulation. In distributed convection, however, there appears no significant modification in the distribution of $\bar{u}_{\theta}$, although large fluctuation associated with convective plumes is superposed (EXP A4 and EXP B4).
We expect much higher lateral buoyancy transfer $\overline{b^{\prime} u_{r}^{\prime}}$ at the surface in localized convection in which the lateral buoyancy transfer is dominantly contributed by baroclinic eddies. This is evidenced in Fig. 9 (EXP A1 and EXP B1) in which $\overline{b^{\prime} u_{r}^{\prime}}$ increases substantially at $r$ $\sim R^{*}$ but its distribution becomes complicated after the breakup of the convective column (after day 6 of EXP A1). Meanwhile, in distributed convection (EXP A4 and EXP B4) the radial buoyancy transfer remains at a very low level, contributed only by small-scale convective eddies.

The lateral buoyancy transfer by baroclinic eddies from the convective column to the outside was suggested by Visbeck et al. (1996) as

$$
\overline{b^{\prime} u_{r}^{\prime}} \sim \frac{\overline{b^{\prime 2}}}{N} .
$$

If buoyancy fluctuation can be estimated by

$$
b^{\prime} \propto \Delta b[=b(r=0)-b(r=\infty)]
$$


(a)

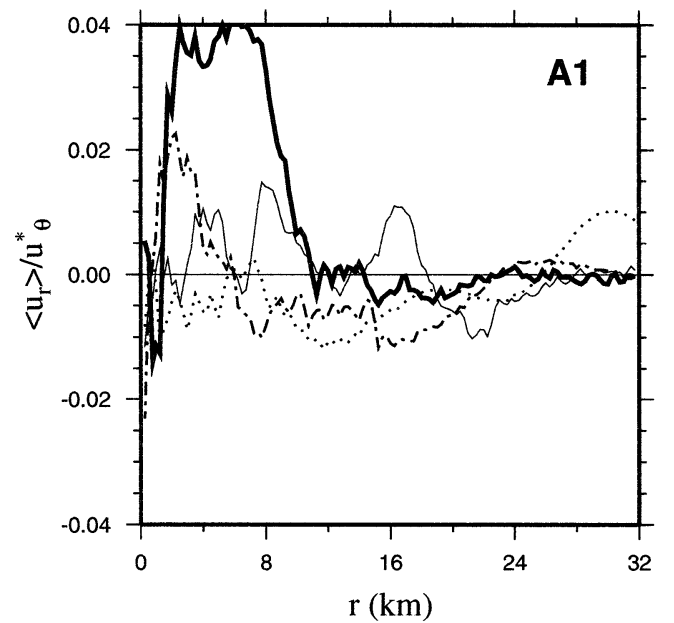

(b)

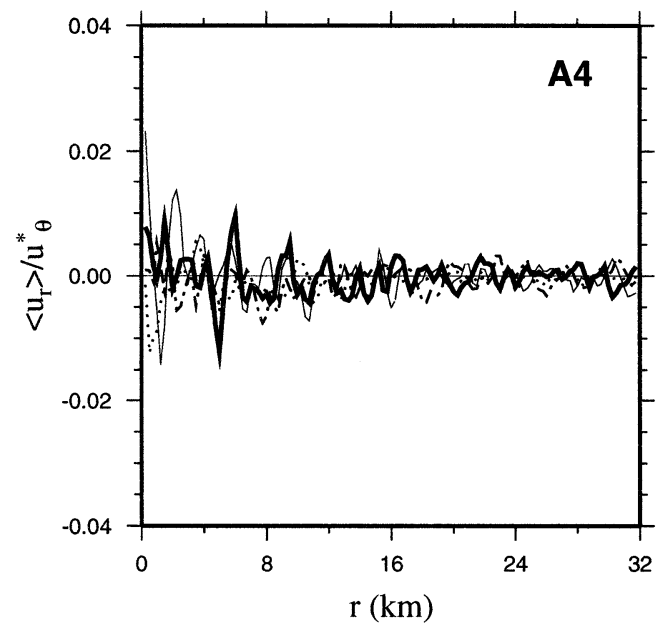

(c)

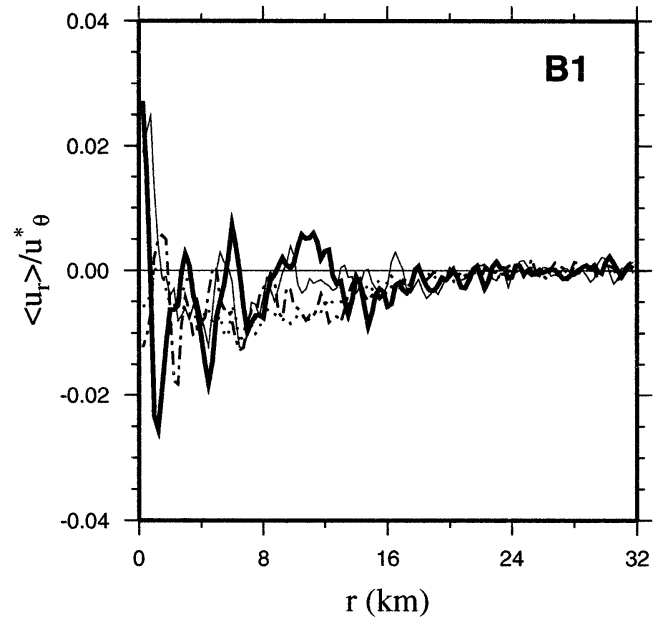

(d)

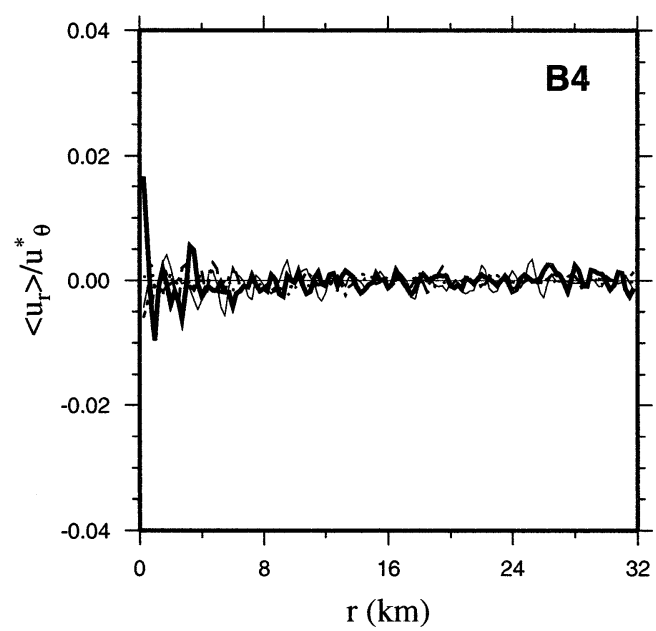

FIG. 7. Evolution of the radial distribution of the azimuthally averaged radial velocity $\bar{u}_{r}$ at the surface scaled by $u_{\theta}^{*}$ (line patterns correspond to days as in Fig. 5) for (a) EXP A1, (b) EXP A4, (c) EXP B1, and (d) EXP B4.

for the present simulation, we can obtain the magnitude of (17) as

$$
\overline{b^{\prime} u_{r}^{\prime}} \propto \frac{(A \gamma)^{2}}{(A \beta)^{1 / 2}} .
$$

The peak values of $\overline{b^{\prime} u_{r}^{\prime}}$ before the breakup of the convective column roughly represent the value estimated from (19) in localized convection, as shown in Fig. 9 (see, e.g., the peaks values on day 2 of EXP A1 and EXP B1). Strong negative buoyancy transfer at day 6 of EXP A1 is related to the strong downdraft near $r \sim$ $R^{*}$, as observed in Fig. 9a.

One should notice, however, that the lateral buoyancy transfer $\overline{b^{\prime} u_{r}^{\prime}}$ does not increase with time, contrary to the case of disk-cooling experiments, since $\Delta b$ decreases with time. This means that the equilibrium state between the lateral buoyancy transfer and the surface buoyancy loss suggested by Visbeck et al. (1996) cannot be reached in preconditioned experiments, as pointed out by Straneo and Kawase (1999).

Last, we mention that the radial buoyancy gradient is found to decrease slowly with time in both cases of localized convection and distributed convection, as we can notice from Figs. 2-4, which is in agreement with Straneo and Kawase (1999) (not shown).

\section{c. The effect of preconditioning on the pattern of convection}

It is now evident that the vigorous cooling event in the ocean may lead to a fundamentally different pattern of convection depending on the preconditioned buoyancy distribution and the associated cyclonic circulation of the ocean, determined by $A$ and $\gamma$.

The clearest distinction between the two different convective regimes is the generation of baroclinic eddies 
(a)

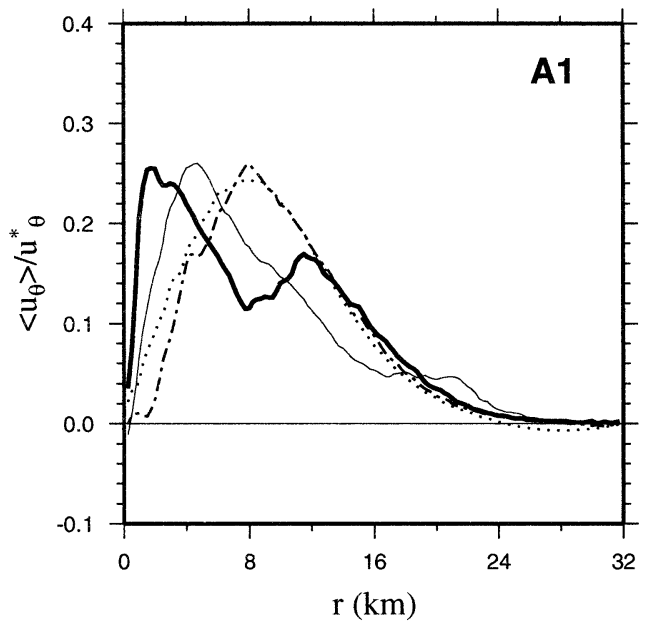

(b)

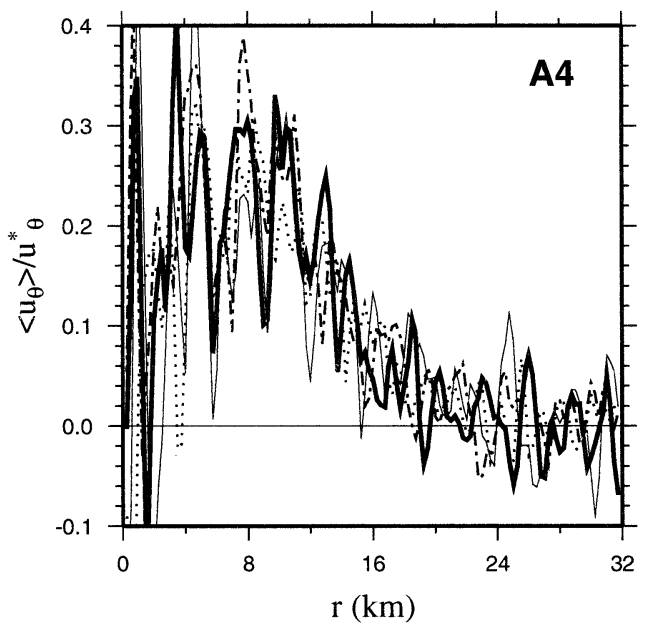

(c)

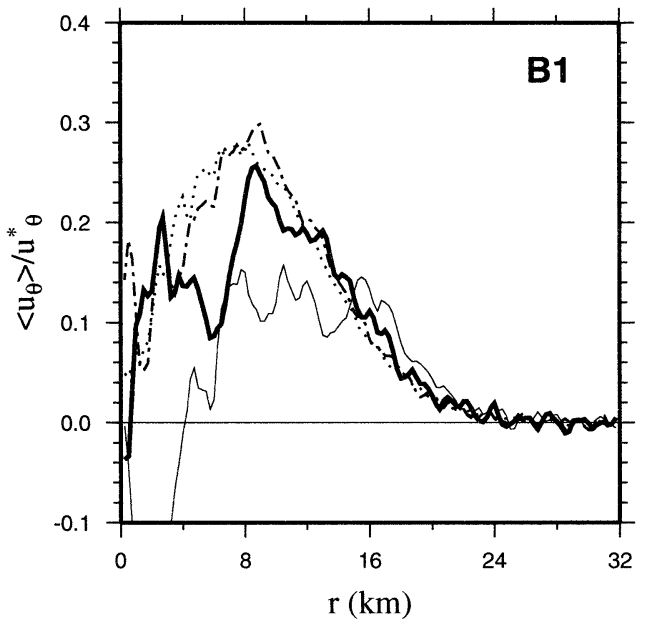

(d)

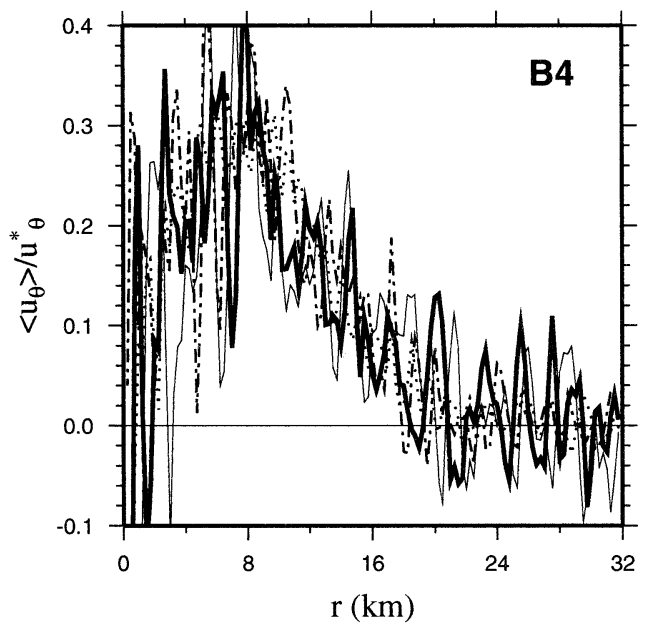

FIG. 8. Evolution of the radial distribution of the azimuthally averaged azimuthal velocity $\bar{u}_{\theta}$ at the surface scaled by $u_{\theta}^{*}$ (line patterns correspond to days as in Fig. 5) for (a) EXP A1, (b) EXP A4, (c) EXP B1, and (d) EXP B4.

that occurs at $r \sim R^{*}$. This can be illustrated from the time series of the difference in horizontal TKE between at $r=R^{*}$ and in the exterior region; that is, $\Delta E_{h}=$ $E_{h}\left(r=R^{*}\right)-E_{h}(r=\infty)$.

If $E_{h}$ is dominated by baroclinic eddies at $r \sim R^{*}$ in localized convection, it should increase with time as

$$
E_{h}\left(r=R^{*}\right) \sim(\sigma t)^{2} \sim \frac{A \gamma^{2}}{\beta R^{2}} t^{2},
$$

according to (16), until the breakup of the convective column. Meanwhile, $E_{h}$ in the exterior region can be scaled by that of the convective boundary layer as (i.e., Denbo and Skyllingstad 1996)

$$
E_{h}(r=\infty) \sim(B h)^{2 / 3} .
$$

Since the energy of convective eddies of (21) is much smaller than the energy of baroclinic eddies of (20) after the initial period in localized convection, as observed in Fig. 5, we can estimate $\Delta E_{h}$ as $\Delta E_{h} \sim E_{h}\left(r=R^{*}\right)$. On the other hand, $E_{h}$ is estimated by (21) both at $r \sim$ $R^{*}$ and in the exterior region in distributed convection. In this case $\Delta E_{h}$ is expected to be very small and does not vary much with time.

Figure 10 shows the time series of $\Delta E_{h}$ for the experiments of EXP A and EXP B. Here the azimuthal average is made over the circular band of thickness of $2 \mathrm{~km}$ around $r=R^{*}$ and $30 \mathrm{~km}$. In localized convection (EXP A1, EXP A2/B1, EXP B2) $\Delta E_{h}$ increases following (20) until the breakup of a convective column. Meanwhile, in distributed convection (EXP A3, EXP A4, EXP B4) the values of $\Delta E_{h}$ are much smaller than those predicted from (20) and do not show noticeable variation with time after the initial stage.

According to the dimensional analysis given by (15), only Ro varies during EXP B, which suggests that the transition from distributed convection to localized con- 
(a)

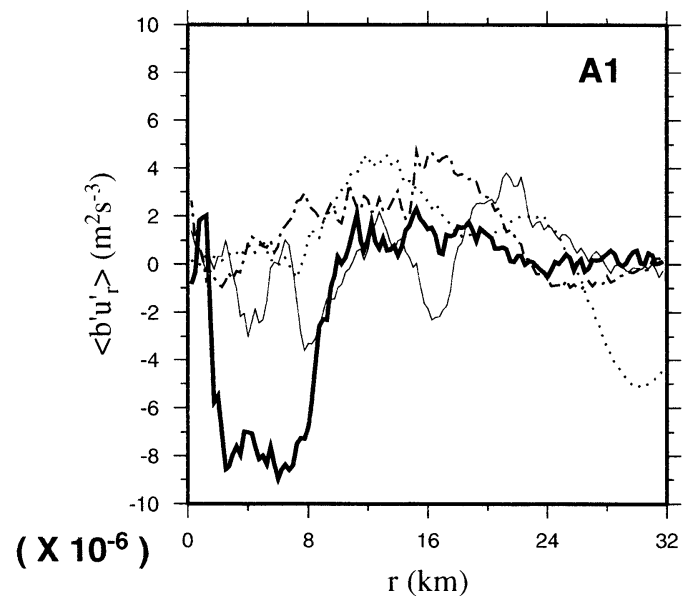

(b)

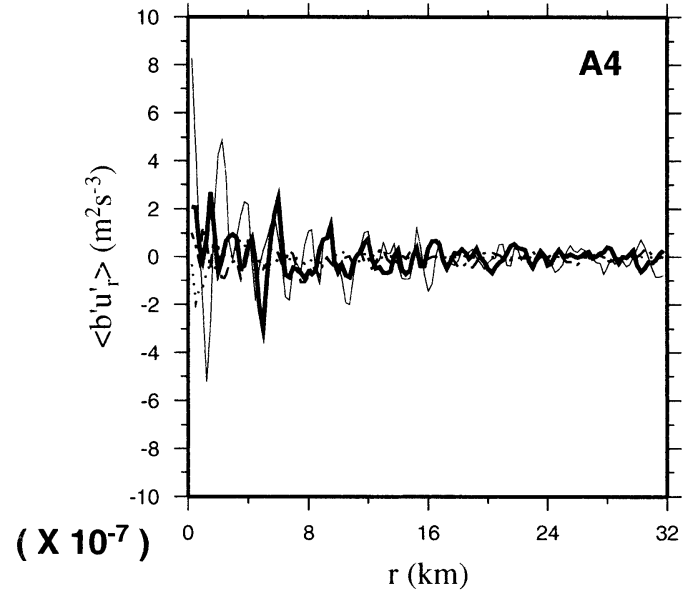

(c)

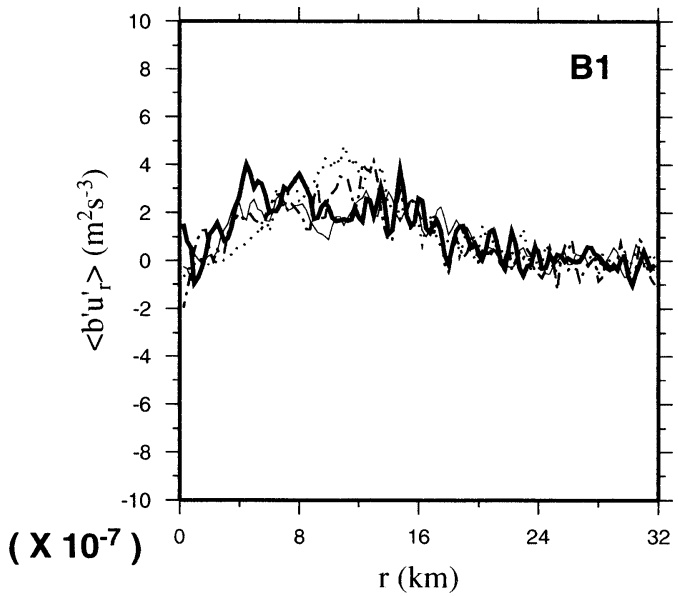

(d)

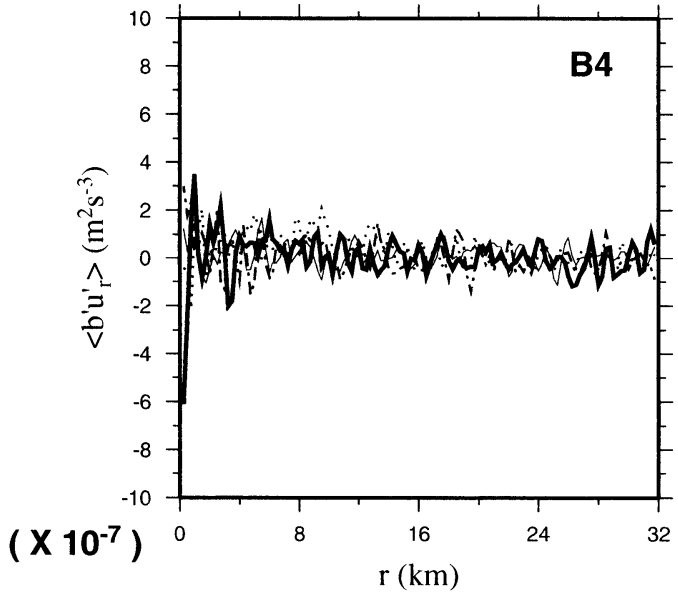

FIG. 9. Evolution of the radial distribution of the radial buoyancy transfer $\overline{b^{\prime} u_{r}^{\prime}}$ at the surface, (line patterns correspond to days as in Fig. 5) for (a) EXP A1, (b) EXP A4, (c) EXP B1, and (d) EXP B4. [Note that the vertical scale of Fig. 9a (EXP A1) is 10 times as large.]

vection occurs with the increase of Ro. The equivalent tendency is also observed from EXP $\mathrm{A}$, although $\mathrm{Bu}$ and Ta also vary during EXP A. The corresponding value of Ro of each experiment is listed in Table 1.

However, it is important to notice that the time series of $\Delta E_{h}$ from EXP B3 is ambiguous to classify. The close examination of other features of convection from EXP B3 also reveals the tendency toward localized convection, although they are dominated by the pattern of distributed convection. This suggests that the transition of the convection pattern may occur rather gradually with intermediate patterns in between.

The radial buoyancy gradient is always degraded after 8 days. It is thus highly unlikely to generate baroclinic instability thereafter, if it does not until that time. Furthermore the cooling event during winter, which generates open-ocean deep convection, usually lasts only a few days. This enhances the significance of the criterion for the convection pattern, shown in Fig. 10.

\section{d. The effects of the intensity of surface cooling}

The results from EXP C, which is the same as EXP A except for the smaller surface buoyancy loss $(B=$ $2.5 \times 10^{-8} \mathrm{~m}^{2} \mathrm{~s}^{-3}$ ), show the similar variation of the convection pattern to EXP A except that the values of $E_{h}, E_{v}, \bar{u}_{r}$, and $\overline{b^{\prime} u_{r}^{\prime}}$ are smaller. Meanwhile, EXP C3 reveals some characteristics of localized convection, although EXP A3 produces distributed convection (not shown). This suggests that the pattern of convection tends to transform from localized convection to distributed convection with increasing $B$.

In order to elucidate the role of the surface buoyancy loss further, we carried out a series of experiments EXP 
(a)

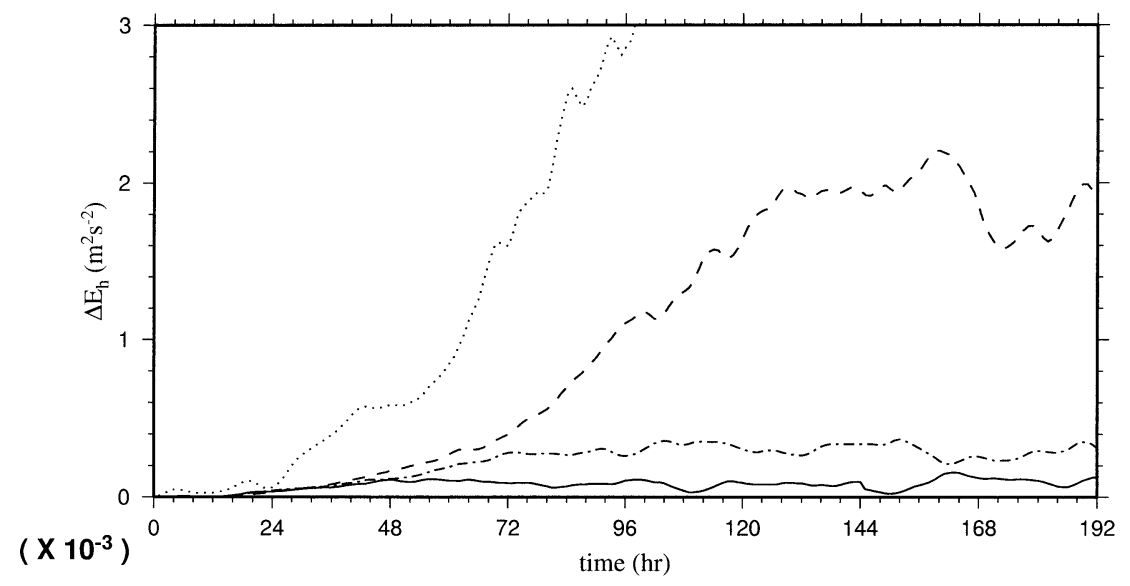

(b)

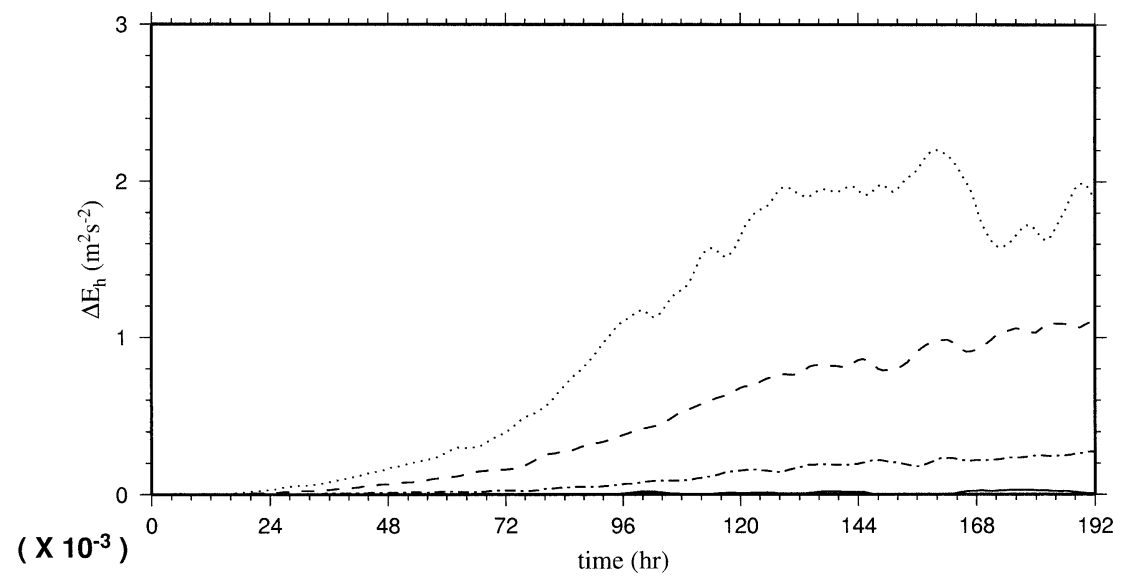

FIG. 10. Time series of $\Delta E_{h}\left[=E_{h}\left(r=R^{*}\right)-E_{h}(r=30 \mathrm{~km})\right]$ at the surface. Here the azimuthal average is made over a circular band of thickness $2 \mathrm{~km}$ for (a) EXP A (dotted: A1; dashed: A2; dot-dashed: A3; solid: A4) and (b) EXP B (dotted: B1; dashed: B2; dot-dashed: B3; solid: B4).

$D$ in which the various values of $B$ are imposed on the same precondition as EXP A2 (or EXP B1). Figures 11-15 show the evolutions of $E_{h}, E_{v}, \bar{u}_{r}, \bar{u}_{\theta}$, and $\overline{b^{\prime} u_{r}^{\prime}}$ from EXP D2 and EXP D5. They show similar contrast between localized convection (EXP D2) and distributed convection (EXP D5), as in the cases of EXP $\mathrm{A}$ and EXP B. One noticeable difference is that a considerable decrease of $\bar{u}_{\theta}$ appears during distributed convection (Fig. 14b), contrary to the cases of EXP A and EXP B (Figs. 8b,d). This means that the radial buoyancy gradient associated with a cyclonic gyre is substantially eroded by strong convective mixing.

We obtained again the times series of $\Delta E_{h}=E_{h}(r=$ $\left.R^{*}\right)-E_{h}(r=\infty)$ from the results of EXP D to differentiate the convection pattern (Fig. 16). It shows that the initial growth of $\Delta E_{h}$ increases with $B$, consistent with Noh et al. (1999) and Yoshikawa et al. (2001). According to Molemaker and Dijkstra (2000), baroclinic instability is initiated as the convective mixed layer reaches to a certain depth for a given preconditioned flow and suggests the faster growth of $\Delta E_{h}$ with increasing $B$. This also reflects the fact that under the stronger cooling the stratification is eroded faster, and thus the smaller $N$ makes $\sigma$ larger in (16).

In the case of EXP D1, $\Delta E_{h}$ was found to keep growing until $t=16$ days. It indicates that baroclinic instability will occur ultimately, once the convective mixed layer reaches to a certain depth, regardless of the intensity of the surface buoyancy loss.

On the other hand, it is observed that $\Delta E_{h}$ stops growing at a certain time and starts to decrease in the cases of EXP D3, D4, and D5. In the case of EXP D3 (or equivalently EXP B1), it is due to the breakdown of the cyclonic gyre (see, e.g., Figs. 2a and 3a), as evidenced by Figs. 5-9. In the cases of EXP D4 and D5, however, it represents that baroclinic eddies decay owing to the erosion of radial buoyancy gradient by convective mixing, as expected from Fig. 14b. Buoyancy distribution at the surface also shows no sign of the breakup of a cyclonic gyre (not shown). As a result, EXP D5 soon 
(a)

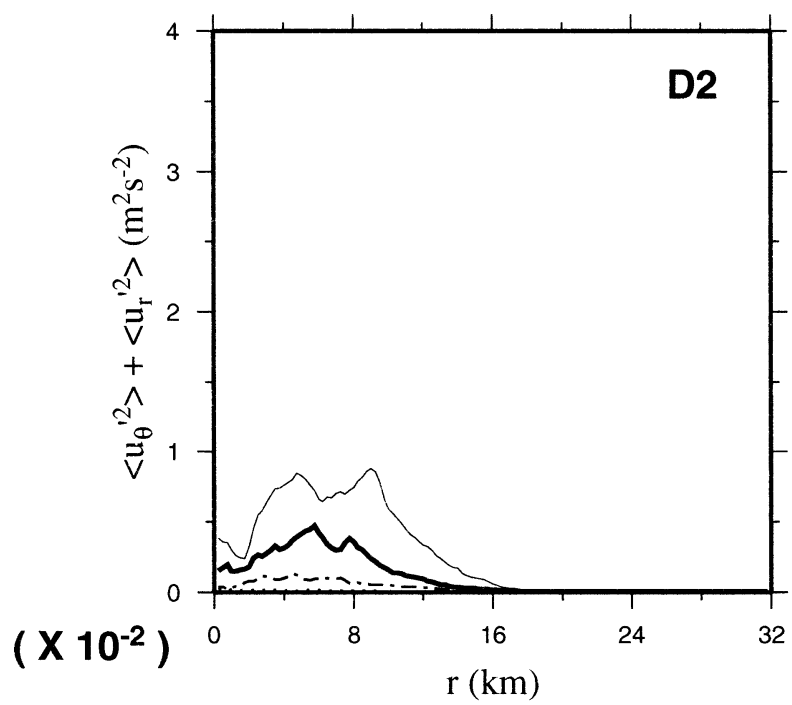

(b)

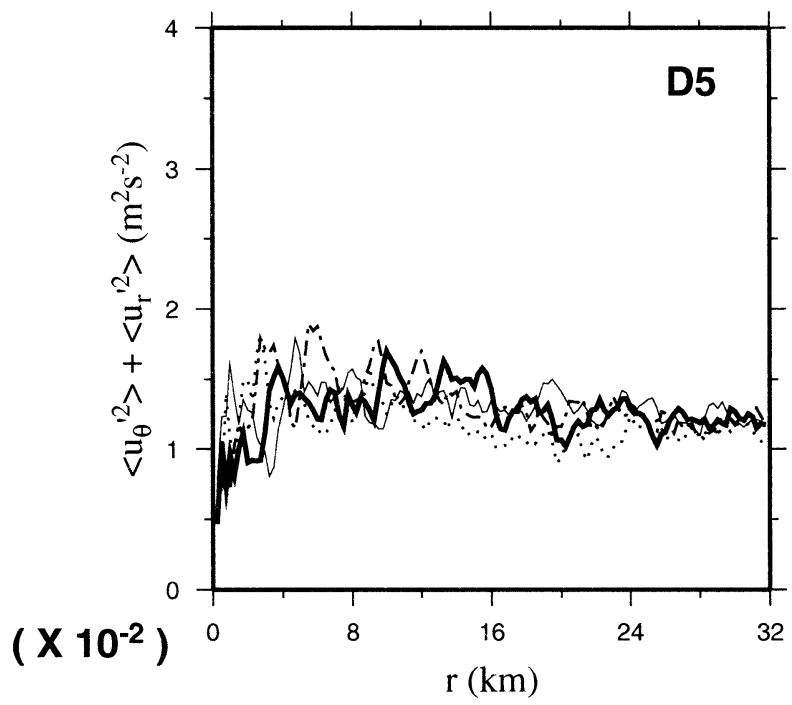

FIG. 11. Evolution of the radial distribution of the azimuthally averaged horizontal turbulent kinetic energy $E_{h}\left[=\left(\overline{u_{r}^{\prime 2}}+\overline{u_{\theta}^{\prime 2}}\right) / 2\right]$ at the surface (dotted line: day 2; dot-dashed: day 4; thick solid: day 6; thin solid: day 8) for (a) EXP D2 and (b) EXP D5.

approaches the convection pattern that can be characterized by distributed convection, as shown in Figs. 1115, while EXP D4 maintains the intermediate pattern during the simulation period.

The results from EXP C and EXP D clearly manifest that the increased surface buoyancy loss inhibits the growth of baroclinic eddies. Two different mechanisms of the inhibition of baroclinic instability by convection are conceivable. One is the increased damping by increasing the eddy viscosity of a flow and the other is the erosion of radial buoyancy gradients. The decay of baroclinic eddies in EXP D4 and D5 after the initial (a)

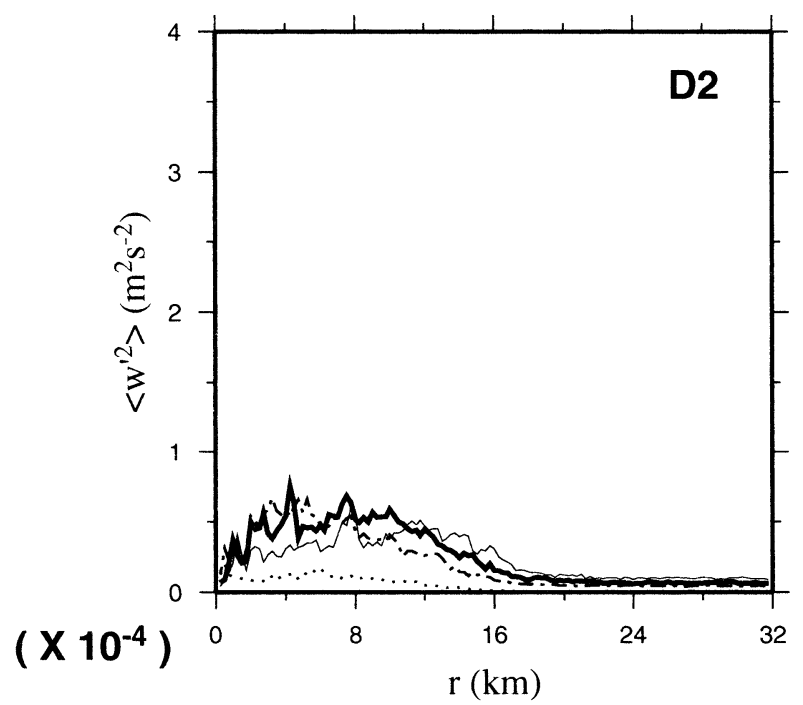

(b)

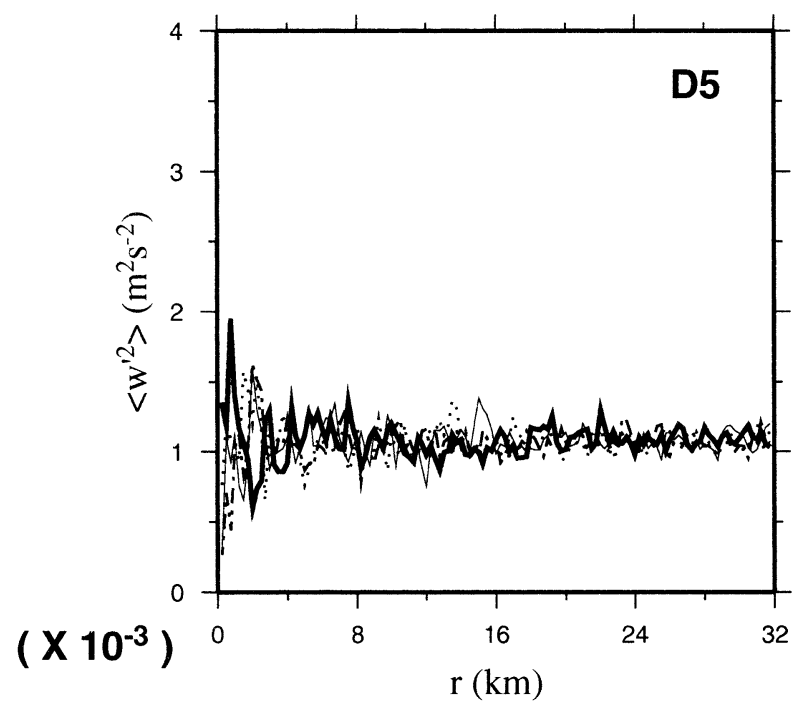

FIG. 12. Evolution of the radial distribution of the azimuthally averaged vertical turbulent kinetic energy $E_{v}\left(=\overline{w^{\prime 2}} / 2\right)$ at $z=100 \mathrm{~m}$ (line patterns correspond to days as in Fig. 11) for (a) EXP D2 and (b) EXP D5. [Note that the vertical scale of Fig. 11b (EXP D5) is 10 times as large.]

growth is certainly affected by the erosion of radial buoyancy gradients. On the other hand, in the cases of EXP A and EXP B the radial buoyancy gradient are not significantly modified, as expected from the distribution of $\bar{u}_{\theta}$ in Fig. 8, despite the occurrence of distributed convection, thus suggesting that the inhibition of baroclinic instability may not be caused by the erosion of the radial buoyancy gradient in the these cases.

With an aim to clarify the situation, we carried out a series of experiments EXP E. During EXP E, only the parameter $B$ varies, as in EXP D, but has the stronger 
(a)

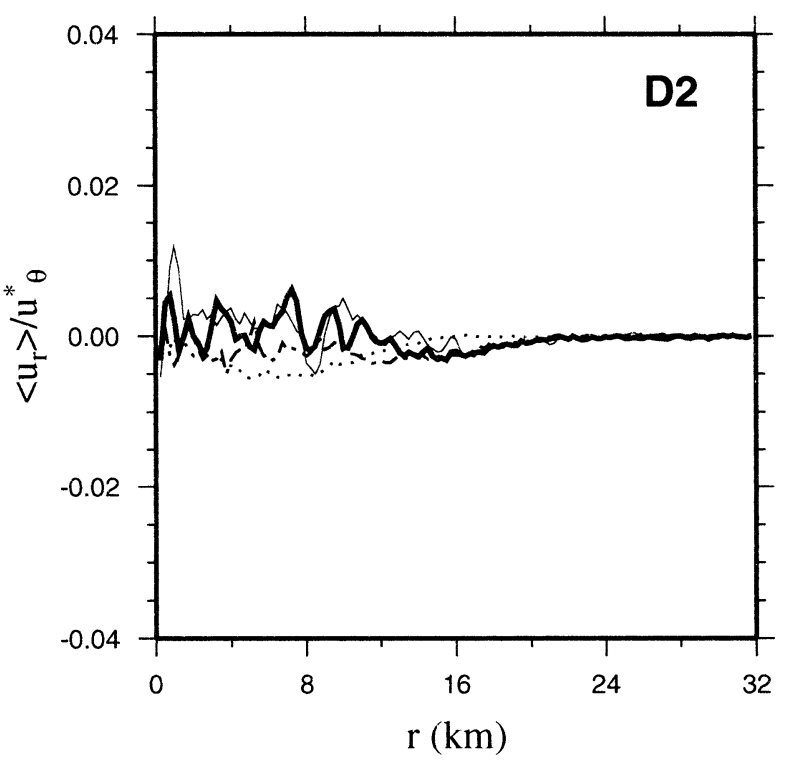

(b)

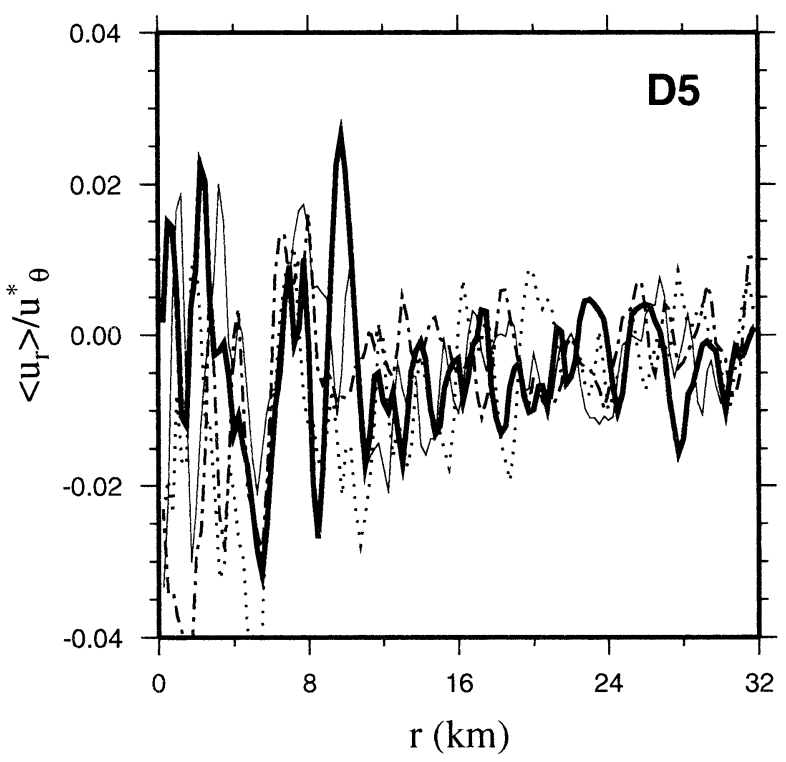

FIG. 13. Evolution of the radial distribution of the azimuthally averaged radial velocity $\bar{u}_{r}$ at the surface scaled by $u_{\theta}^{*}$ (line patterns correspond to days as in Fig. 11) for (a) EXP D2 and (b) EXP D5.

background stratification and a weaker cyclonic gyre (see Table 1). In these experiments the contact of the convective mixed layer with the bottom, which modifies the radial buoyancy gradient seriously, can be avoided, and the preconditioning is less susceptible to baroclinic instability than EXP D. Moreover, in this case the growth rate of baroclinic eddies $\sigma$, given by (16), remains the same, unlike the cases of EXP A and EXP B.

One leads to localized convection (EXP E1) and the other leads to distributed convection (EXP E2). Figure (a)

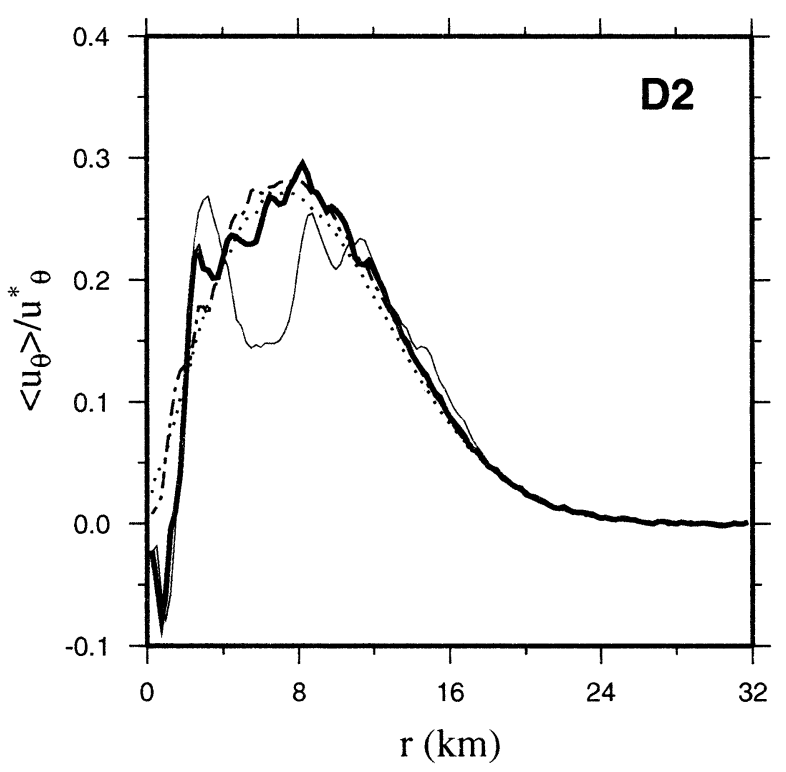

(b)

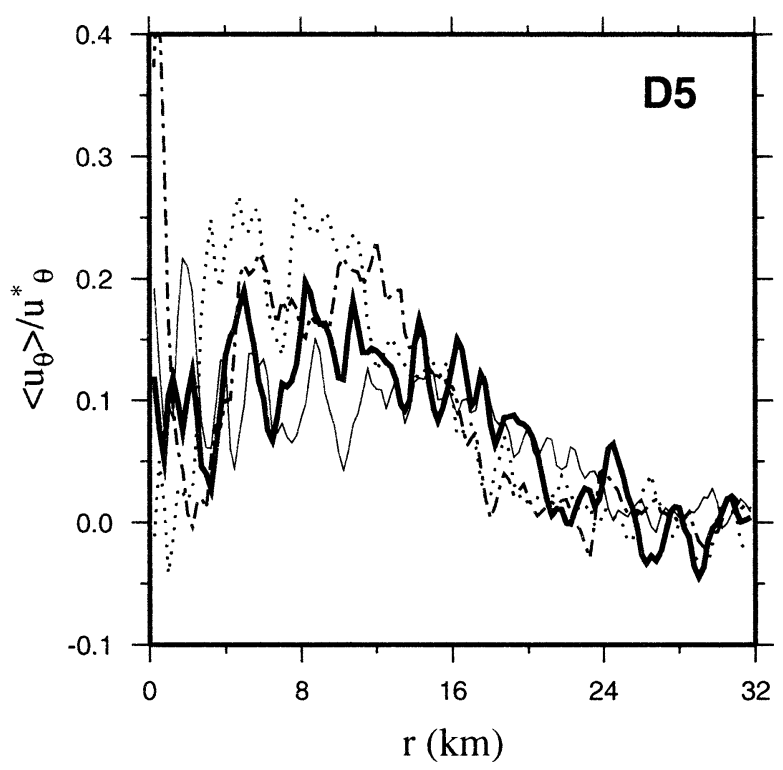

FIG. 14. Evolution of the radial distribution of the azimuthally averaged azimuthal velocity $\bar{u}_{\theta}$ at the surface scaled by $u_{\theta}^{*}$ (line patterns correspond to days as in Fig. 11) for (a) EXP D2 and (b) EXP D5.

17 shows that baroclinic instability is generated in EXP E1 but not in EXP E2. Here the $x$ axis $B t$ represents the total amount of buoyancy loss since the start of cooling, which determines the nonpenetrative deepening of the convective mixed layer [see, e.g., (6)] and consequently the generation of baroclinic instability (Molemaker and Dijkstra 2000). However, the radial buoyancy gradients from both experiments are almost identical at this stage (Fig. 18), although the values of buoyancy are slightly 
larger in EXP E2 because of stronger entrainment. This clearly shows that the inhibition of baroclinic instability is not caused by the erosion of radial buoyancy gradient in this case, but rather by the increased damping under stronger convection.

Consequently, we can presume that the inhibition of baroclinic instability is primarily caused by the increased damping under stronger convection (EXP A, EXP B, and EXP G), although the erosion of radial buoyancy gradient plays an important role in certain situations for the decay of baroclinic instability (EXP D). Note also that, according to Molemaker and Dijkstra (2000), baroclinic instability usually occurs at the very early stage of convective deepening, that is, $h \beta \sim 0.01$, at which the erosion of radial buoyancy gradients is insignificant.

\section{e. A regime diagram for the classification of convection}

The analyses of the simulation results so far showed that it tends to become localized convection as the intensity of a cyclonic gyre increases and the surface buoyancy loss decreases, or equivalently with the increases of Ro and Ta according to the dimensional analysis of (15). Figure 19 shows the regime diagram to classify the convection pattern in terms of Ro and Ta, based on the experimental results listed in Table 1 .

Note, however, that there still remains another nondimensional parameter $\mathrm{Bu}$ in (15) that may affect the convection pattern. Therefore, we examined the effects of $\mathrm{Bu}$ in EXP $\mathrm{F}$ and EXP $\mathrm{G}$ in which only $\mathrm{Bu}$ varies while Ro and Ta are fixed.

The results from EXP $\mathrm{F}$ show that the convection pattern is not significantly affected by the change of $\mathrm{Bu}$. For example, in the time series of $\Delta E_{h}=E_{h}\left(r=R^{*}\right)$ $-E_{h}(r=\infty)$ every experiment of EXP F represents the growth of baroclinic eddies associated with localized convection, although its growth rate tends to decrease slightly with the increase of $B$ (Fig. 20). Note that EXP $\mathrm{F}$ covers almost the maximum range of $\mathrm{Bu}$ variation, corresponding to $\gamma=0.1-0.9$. We also found that the pattern of distributed convection also remains unaffected by the change of $\mathrm{Bu}$ in EXP G.

This suggests that the primary role of background stratification is to counteract the convective mixing; therefore its contribution is largely represented by Ta. It means that one can refer the convection pattern from the regime diagram of Fig. 19 in most cases. It is also worth mentioning that the generation of baroclinic instability in a rotating annulus of fluid subjected to differential heating is determined by Ro and Ta in a similar fashion (Fowlis and Hide 1965; Tritton and Davis 1985).

Note, however, that, if we plot a regime diagram in terms of Ro and $B / f^{3} R^{2}$, based on the dimensional analysis given by (10), the results strongly depend on $\mathrm{Bu}$ that is the only parameter representing the background (a)

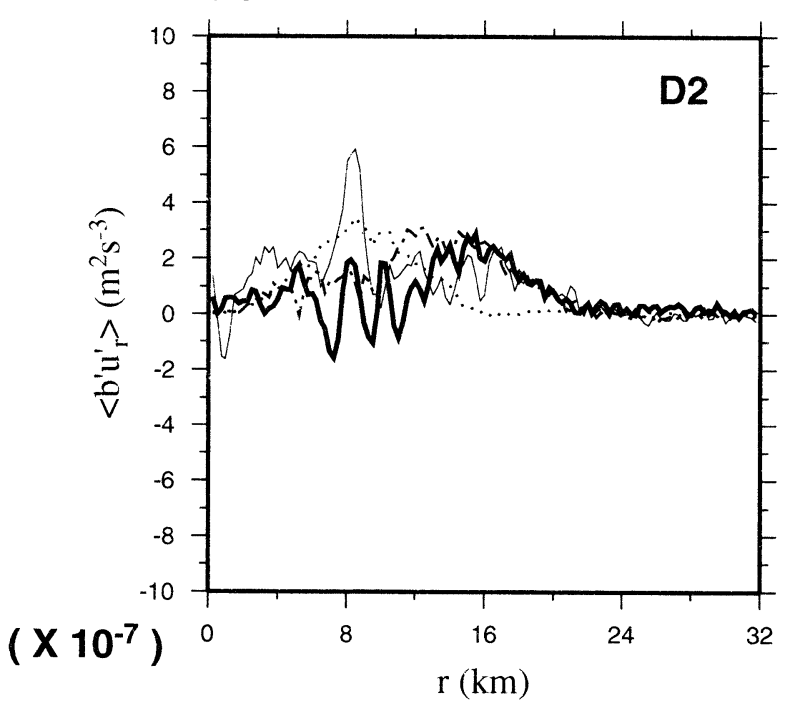

(b)

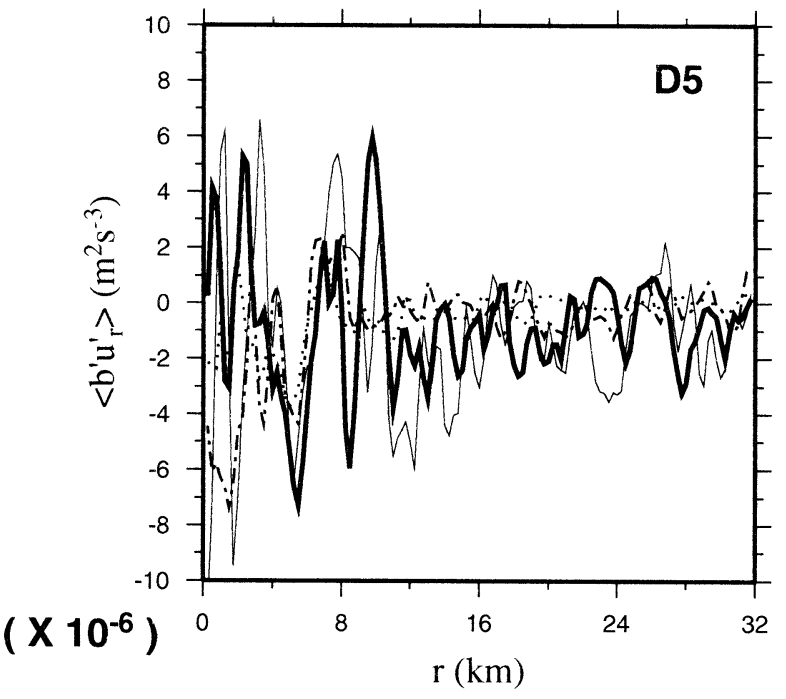

FIG. 15. Evolution of the radial distribution of the radial buoyancy transfer $\overline{b^{\prime} u_{r}^{\prime}}$ at the surface (line patterns correspond to days as in Fig. 11) for (a) EXP D2 and (b) EXP D5. [Note that the vertical scale of Fig. 15b (EXP D5) is 10 times as large.]

stratification in (10), and two regimes are not clearly divided in the Ro $-B / f^{3} R^{2}$ domain.

\section{Summary and discussion}

In this paper we have shown that open-ocean deep convection occurs in a fundamentally different pattern, depending on the precondition of the ocean, by analyzing the results from large eddy simulation.

As the intensity of a cyclonic gyre in the ocean increases, the typical pattern of open-ocean deep convection appears, such as the generation of baroclinic instability, large lateral buoyancy transfer, secondary cir- 


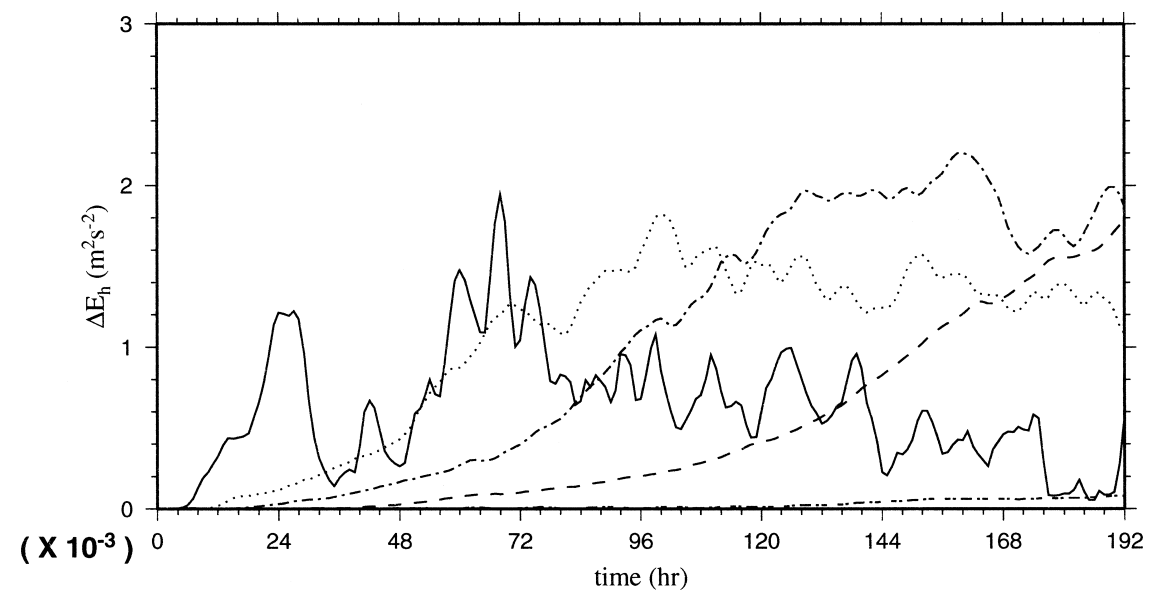

FIG. 16. Time series of $\Delta E_{h}\left[=E_{h}\left(r=R^{*}\right)-E_{h}(r=30 \mathrm{~km})\right]$ at the surface from EXP D. Here the azimuthal average is made over a circular band of thickness $2 \mathrm{~km}$ (dot-short/long dashed: D1; dashed: D2; dot-dashed: D3; dotted: D4; solid: D5)

culation, restratification, and the breakup of the original cyclonic gyre (localized convection). On the other hand, as the intensity of a cyclonic gyre decreases, small-scale convective plumes appear uniformly over the whole surface similarly to the convective boundary layer without generating the typical features of open-ocean deep convection mentioned above (distributed convection).

It is also found that the transition from distributed convection to localized convection is inhibited as the surface buoyancy loss increases. It is likely due to the increased damping under stronger convection, although the erosion of radial buoyancy gradient plays an important role in certain situations.

Based on dimensional analysis, we could obtain the parameters that determine the pattern of convection as $\mathrm{Ro}, \mathrm{Ta}$, and $\mathrm{Bu}$, which are defined by $\mathrm{Ro}=u_{\theta}^{*} / f R$, Ta $=\left[\left(B / N_{0}^{2}\right) / f H^{2}\right]^{-2}$, and $\mathrm{Bu}=N_{0} H / f R$. Here, $N_{0}$ is the Brunt-Väisälä frequency at the surface, $R$ and $H$ are the horizontal and vertical scales of a cyclonic gyre, $f$ is the Coriolis frequency, $B$ is the surface buoyancy loss, and $u_{\theta}^{*}$ is the velocity scale of the gyre (see section 3 ). It was found that the transition to localized convection from distributed convection occurs with increasing Ro and $\mathrm{Ta}$, but it is insensitive to $\mathrm{Bu}$. A regime diagram to predict the convection pattern in terms of Ro and Ta is presented (Fig. 19).

According to the present results, we are able to predict various important aspects of open-ocean deep convection, once the precondition of the ocean and the magnitude of the surface buoyancy flux are known: for example, whether restratification in the core of deep convection will occur or how much lateral heat transfer will be induced during convection. Furthermore, the enormous difference in the energy of generated baroclinic eddies, or eddy kinetic energy, between localized convection and distributed convection will have the important implication in understanding and predicting the ocean circulation.

Meanwhile, it is important to mention that the criterion suggested in this paper helps us to predict how

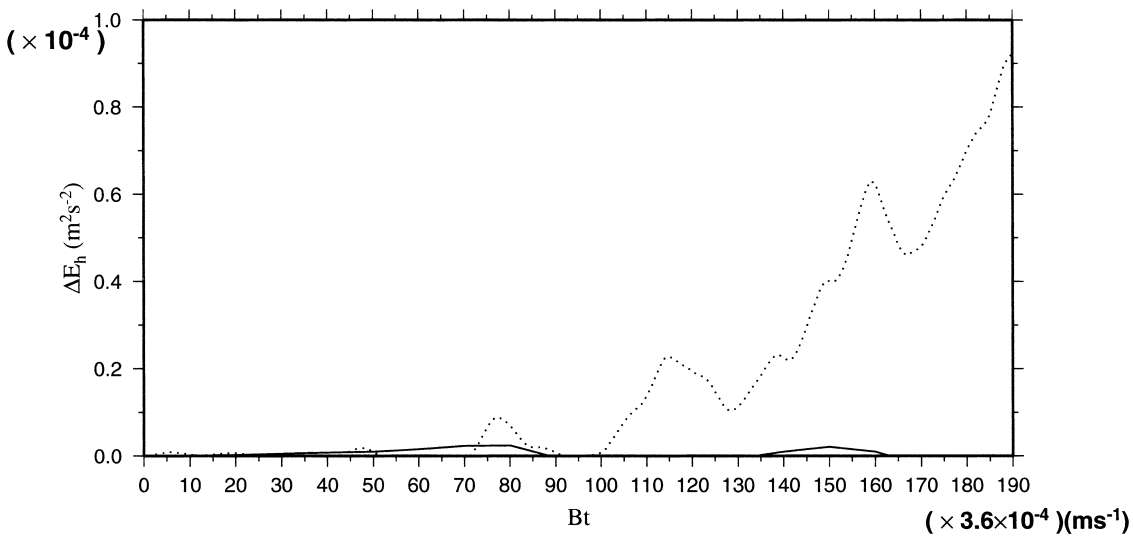

FIG. 17. Time series of $\Delta E_{h}\left[=E_{h}\left(r=R^{*}\right)-E_{h}(r=30 \mathrm{~km})\right]$ at the surface from EXP E (dotted: E1; solid: E2). Here the azimuthal average is made over a circular band of thickness 2 $\mathrm{km}$. Note that the $x$ axis indicates $B t$ in this case. 


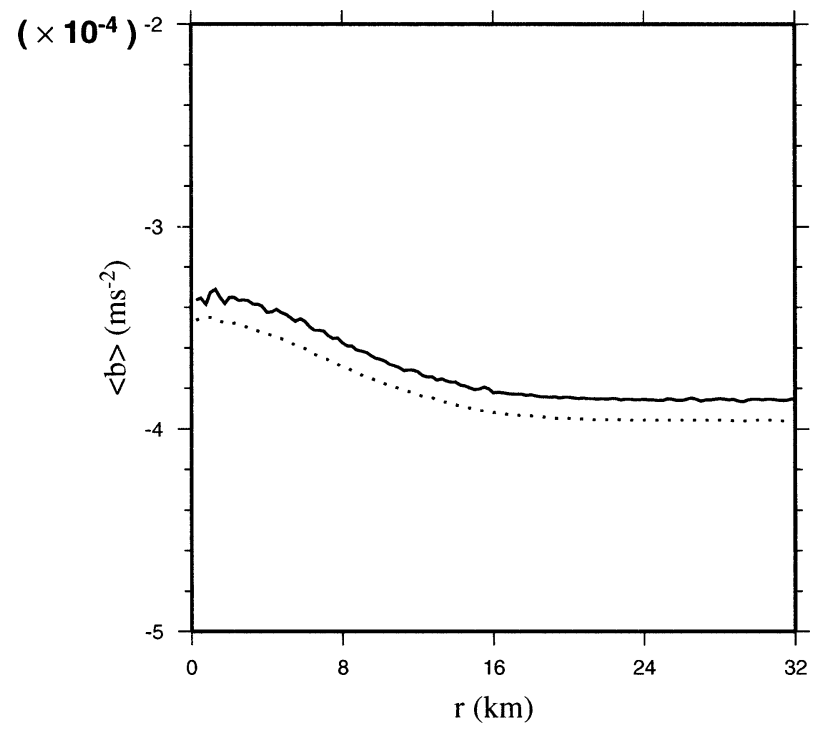

FIG. 18. The radial distribution of the azimuthally averaged buoyancy at $B t=5.4 \times 10^{-2} \mathrm{~m}^{2} \mathrm{~s}^{-2}$ (dotted: E1; solid: E2).

convection evolves, not whether convection can penetrate into the deep ocean. The preconditioning for localized convection appears to be favorable to deeper penetration with its stronger isopycnal domic structure. However, lateral mixing and restratification, which suppress the penetration into the deep ocean, are absent in distributed convection.

The parameters considered in the paper with regard to the preconditioning of the ocean are relatively well documented in the case of the northwestern Mediterranean deep convection (Schott et al. 1994; Marshall and Schott 1999). The maximum rim current velocity around the convection patch was estimated as $u_{\theta}^{*} \sim 15$ $\mathrm{cm} \mathrm{s}^{-1}$. The patch size was observed as $20-30 \mathrm{~km}$, from which we can estimate that $R^{*} \sim 10-15 \mathrm{~km}$. This sug-

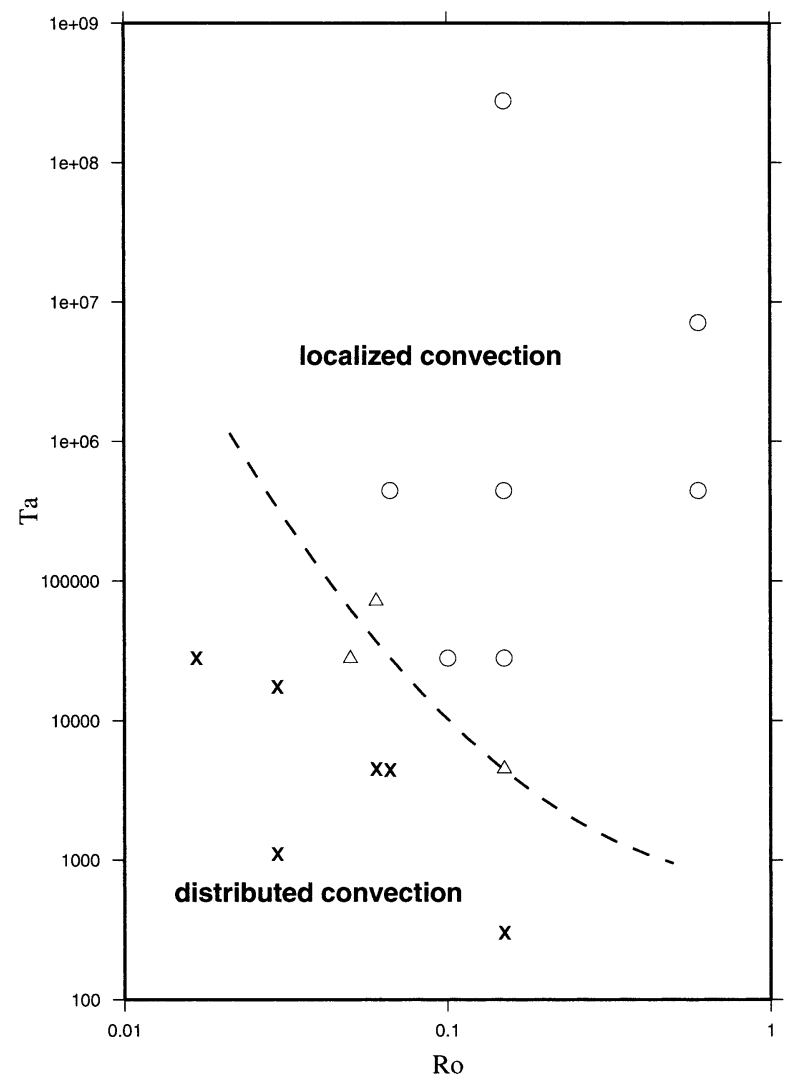

FIG. 19. A regime diagram for the classification of convection pattern (open circles: localized convection; crosses: distributed convection; open triangles: the intermediate pattern).

gests that Ro $\sim 0.1$. Meanwhile, taking the typical values for the surface stratification outside the convection patch and the surface buoyancy loss as $N_{0} \sim 2 \times 10^{-3}$ $\mathrm{s}^{-1}$ and $B \sim 2 \times 10^{-7} \mathrm{~m}^{2} \mathrm{~s}^{-3}$, we can estimate that Ta $\sim 10^{6}$. This suggests that the deep convection at the

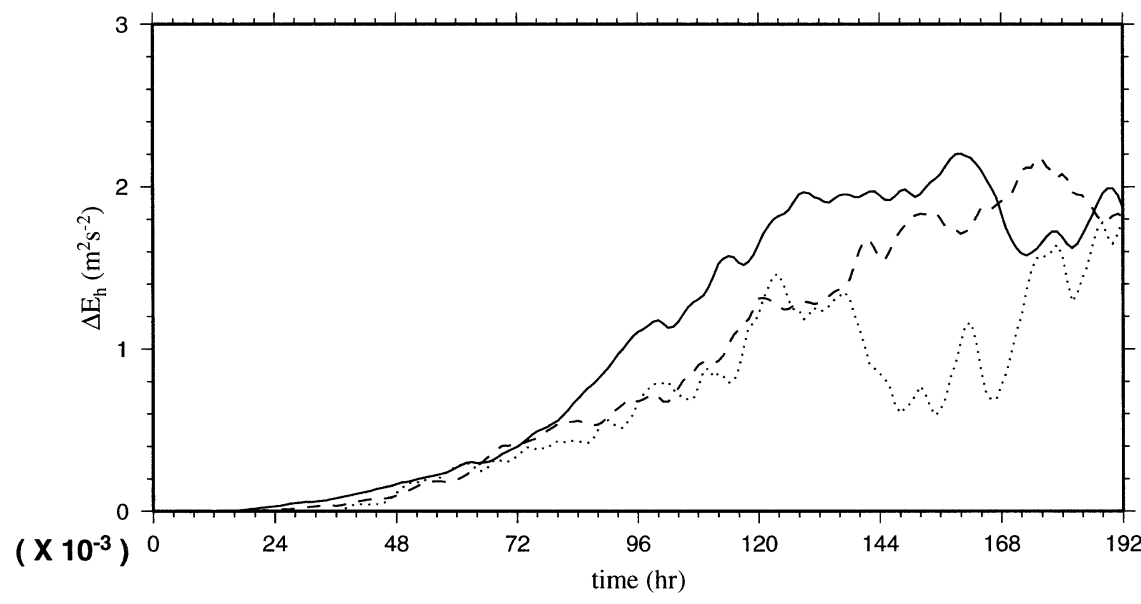

FIG. 20. Time series of $\Delta E_{h}\left[=E_{h}\left(r=R^{*}\right)-E_{h}(r=30 \mathrm{~km})\right]$ at the surface from EXP F. Here the azimuthal average is made over a circular band of thickness $2 \mathrm{~km}$ (solid: F1; dashed: F2; dotted: F3). 
northwestern Mediterranean may occur in the form of localized convection, according the regime diagram of Fig. 19. The presence of baroclinic eddies (Gascard 1978) and restratification may confirm the occurrence of localized convection.

We hope that more systematic analysis of the observation data, including the open-ocean convection in the Labrador Sea and the Greenland Sea, will clarify the relation between the preconditioning and the pattern of convection in the future research. Further, it will be interesting to investigate how the results are affected by other factors such as ice, thermobaricity, and the size of a gyre.

Last, we have found that the simulation with constant eddy viscosity and diffusivity $\left(\nu_{T}=\kappa_{T}=0.7 \mathrm{~m}^{2} \mathrm{~s}^{-1}\right)$ tends to inhibit the generation of baroclinic instability with a well-defined front between the convective core and the outer region and to erode the stratification below the convective mixed layer. More detailed investigation may be required in the future research for the proper parameterization of the subgrid-scale turbulence in the simulation.

Acknowledgments. This work was supported by Korea/Japan Joint Research Project of KOSEF, the Climate Environment System Research Center sponsored by the SRC program of KOSEF, and the Ecotechnopia Project by KIEST.

\section{REFERENCES}

Asselin, R., 1972: Frequency filter for time integrations. Mon. Wea. Rev., 100, 487-490.

Coates, M. J., G. N. Ivey, and J. R. Taylor, 1995: Unsteady, turbulent convection into a rotating, linearly stratified fluid: Modeling deep ocean convection. J. Phys. Oceanogr., 25, 3032-3050.

Deardorff, J. W., 1980: Stratocumulus-capped mixed layers derived from a three-dimensional model. Bound.-Layer Meteor., 18, 495-572.

Denbo, D. W., and E. D. Skyllingstad, 1996: An ocean large-eddy simulation model with application to deep convection in the Greenland Sea. J. Geophys. Res., 101, 1095-1110.

Eady, E. T., 1949: Long wave and cyclonic waves. Tellus, 1, 513547.

Fowlis, W. W., and R. Hide, 1965: Thermal convection in a rotating annulus of liquid: Effect of viscosity on the transition between axisymmetric and non-axisymmetric flow regimes. J. Atmos. Sci., 22, 541-558.

Gascard, J.-C., 1978: Mediterranean deep water formation, baroclinic eddies and ocean eddies. Oceanol. Acta, 1, 313-315.

Griffiths, R. W., and P. F. Linden, 1981: The stability of vortices in a rotating, stratified fluid. J. Fluid Mech., 105, 283-319.

Hide, R., 1958: An experimental study of thermal convection in a rotating liquid. Philos. Trans. Roy. Soc. London, 250A, 441478.

Ivey, G. N., J. R. Taylor, and M. J. Coates, 1995: Convectively driven mixed layer growth in a rotating, stratified fluid. Deep-Sea Res., 42, 331-339.

Jones, H., and J. Marshall, 1993: Convection with rotation in a natural ocean: A study of open-ocean convection. J. Phys. Oceanogr., 23, 1009-1039.

Legg, S., and J. McWilliams, 2000: Temperature and salinity variability in heterogeneous ocean convection. J. Phys. Oceanogr., 30, 1188-1206.

— - , and J. Gao, 1998: Localization of deep ocean convection by a mesoscale eddy. J. Phys. Oceanogr., 28, 944-970.

Madec, G., M. Chartier, P. Delecluse, and M. Crepon, 1991: A threedimensional numerical model of deep-water formation in the northwestern Mediterranean Sea. J. Phys. Oceanogr., 21, 13491371.

Marshall, J., and F. Schott, 1999: Open-ocean convection: Observations, theory, and models. Rev. Geophys., 37, 1-64.

Maxworthy, T., and S. Narimousa, 1994: Unsteady turbulent convection into a homogeneous, rotating fluid, with oceanographic applications. J. Phys. Oceanogr., 24, 865-887.

Molemaker, M., and H. A. Dijkstra, 2000: Stability of a cold core eddy in the presence of convection: Hydrostatic versus nonhydrostatic modeling. J. Phys. Oceanogr., 30, 475-494

Noh, Y., C. J. Jang, and J. W. Kim, 1999: Large eddy simulation of open ocean deep convection with application to the deep water formation in the East Sea (Japan Sea). J. Oceanogr., 55, 347367.

Pedlosky, J., 1985: The instability of continuous heton clouds. $J$. Atmos. Sci., 42, 1477-1486.

Piacsek, S. A., and G. P. Williams, 1970: Conservation properties of convection difference schemes. J. Appl. Meteor., 9, 856-861.

Raasch, S., and D. Etling, 1991: Numerical simulation of rotating turbulent thermal convection. Beitr. Phys. Atmos., 64, 185-199.

_ and _ 1998: Modeling deep ocean convection: Large eddy simulation in comparison with laboratory experiments. J. Phys. Oceanogr., 28, 1786-1802.

— , and M. Schröter, 2001: PALM - A large eddy simulation model performing on massively parallel computers. Z. Meteor., 10, 363-372.

Sander, J., D. Wolf-Gladrow, and D. Olbers, 1995: Numerical studies of open-ocean deep convection. J. Geophys. Res., 100, 20579 20600.

Saunders, P. M., 1973: The instability of a baroclinic gyre. J. Phys. Oceanogr., 3, 61-65.

Schott, F., M. Visbeck, and U. Send, 1994: Open ocean deep convection: Mediterranean and Greenland Seas. Ocean Processes in Climate Dynamics: Global and Mediterranean Examples, P. Malanotte-Rizzoli and A. R. Robinson, Eds., Kluwer Academic, 203-220.

Send, U., and R. Käse, 1998: Parameterization of process in deep convection regimes. Ocean Modeling and Parameterization, E. P. Chassignet and J. Verron, Eds., Kluwer Academic, 191-214.

Straneo, F., and M. Kawase, 1999: Comparison of localized convection due to localized forcing and to preconditioning. J. Phys. Oceanogr., 29, 55-68.

Tritton, D. J., and P. A. Davies, 1985: Instability of geophysical fluid dynamics. Hydrodynamic Instability and the Transition to Turbulence, H. L. Swinney and J. P. Gollub, Eds., Springer Verlag, 229-270.

Turner, J. S., 1973: Buoyancy Effects in Fluids. Cambridge University Press, 368 pp.

Visbeck, M., J. Marshall, and H. Jones, 1996: Dynamics of isolated convective regions in the ocean. J. Phys. Oceanogr., 26, 17211734.

Whitehead, J. A., J. Marshall, and G. E. Hufford, 1996: Localized convection in rotating, stratified fluid. J. Geophys. Res., 101, $25705-25721$.

Yoshikawa, Y., K. Akimoto, and T. Awaji, 2001: Formation process of intermediate water in baroclinic current under cooling. J. Geophys. Res., 106, 1033-1051. 